\title{
The Adaptive Dynamics of Altruism in Spatially Heterogeneous Populations
}

Jean-Francois Le Galliard (galliard@biologie.ens.fr) Régis Ferrière (regis.ferriere@snv.jussieu.fr) Ulf Dieckmann (dieckman@iiasa.ac.at)

\section{Approved by}

Leen Hordijk (hordijk@iiasa.ac.at)

Director

January 2003 Institute, its National Member Organizations, or other organizations supporting the work. 


\section{Contents}

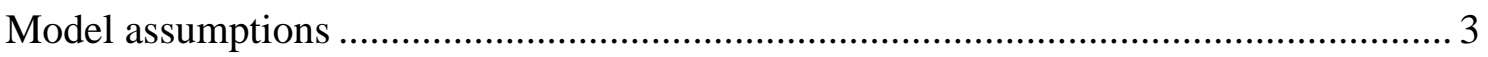

Spatial population dynamics of residents .............................................................. 5

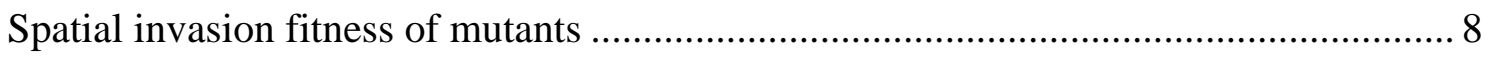

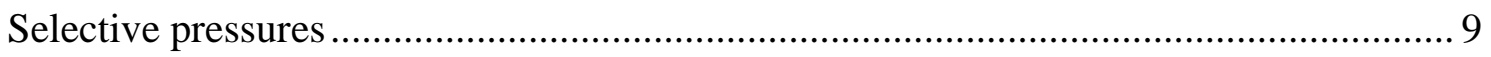

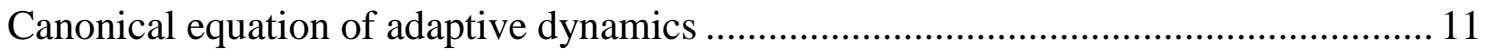

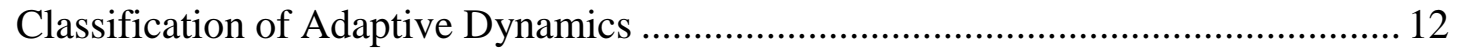

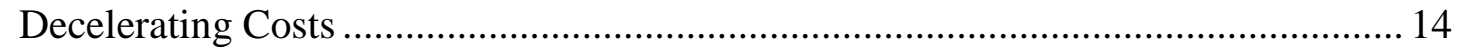

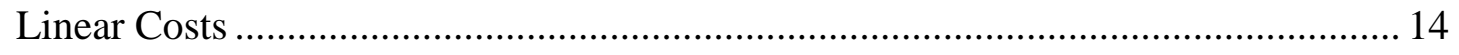

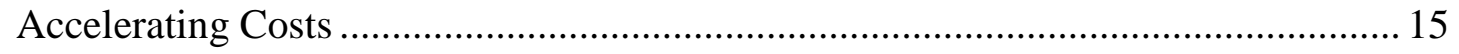

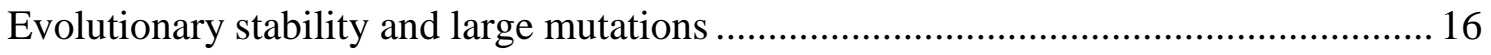

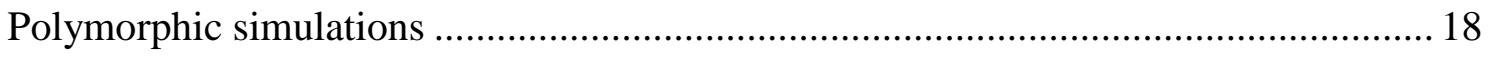

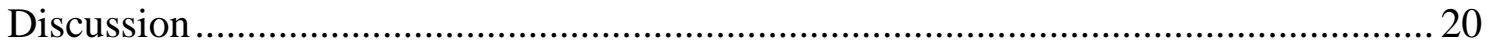

Kin Selection, Cooperation and Competition ......................................................... 21

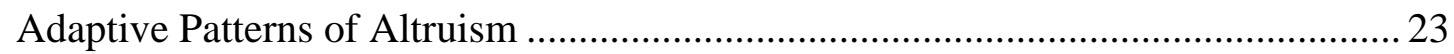

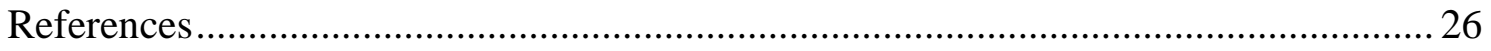

Appendix 1 - Polymorphic individual-based model ….................................................. 30

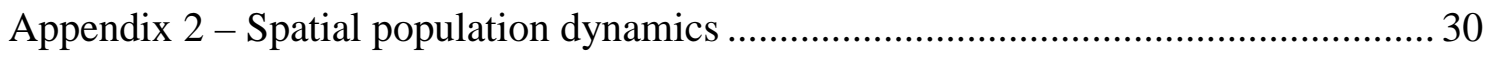

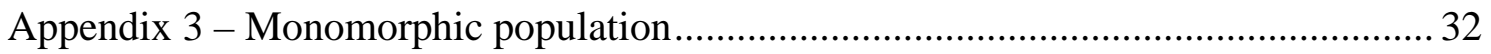

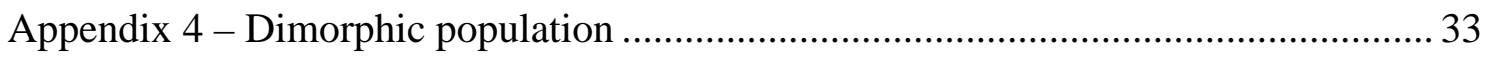

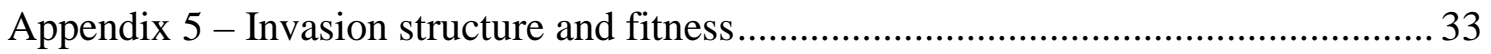




\begin{abstract}
We study the spatial adaptive dynamics of a continuous trait that measures individual investment in altruism. Our study is based on an ecological model of a spatially heterogeneous population from which we derive an appropriate measure of fitness. The analysis of this fitness measure uncovers three different selective processes controlling the evolution of altruism: the direct physiological cost, the indirect genetic benefits of cooperative interactions, and the indirect genetic costs of competition for space. In contrast with earliest suggestions, we find that the cost of competing for space with relatives exerts a negligible selective pressure against altruism. Our study yields a classification of adaptive patterns of altruism according to how the costs of altruism vary with an individual's investment in altruism (we distinguish between decelerating, linear, and accelerating dependence). The invasion of altruism occurs readily in species with accelerating costs, but large mutations are critical for altruism to evolve in selfish species with decelerating costs. Strict selfishness is maintained by natural selection only under very restricted conditions. In species with rapidly accelerating costs, adaptation leads to an evolutionarily stable rate of investment in altruism that decreases smoothly with the level of mobility. A rather different adaptive pattern emerges in species with slowly accelerating costs: high altruism evolves at low mobility, whereas a quasi-selfish state is promoted in more mobile species. The high adaptive level of altruism can be predicted solely from habitat connectedness and physiological parameters that characterize the pattern of cost. We also show that environmental changes that cause increased mobility in those highly altruistic species can beget selection-driven selfextinction, which may contribute to the rarity of social species.
\end{abstract}




\title{
About the Authors
}

\author{
Jean-Francois Le Galliard \\ CNRS UMR 7625 \\ Ecole Normale Supérieure \\ 46 rue d'Ulm \\ 75005 Paris, France \\ Régis Ferrière \\ CNRS UMR 7625 \\ Ecole Normale Supérieure \\ 75005 Paris, France \\ and \\ Adaptive Dynamics Network \\ International Institute for Applied Systems Analysis \\ A-2361 Laxenburg, Austria \\ and \\ University of Arizona \\ Tucson AZ 85721, USA \\ Ulf Dieckmann \\ Adaptive Dynamics Network \\ International Institute for Applied Systems Analysis \\ A-2361 Laxenburg, Austria
}

\section{Acknowledgements}

We thank Y. Iwasa for pointing out the contrast between fecundity altruism and survival altruism. We are grateful to M. van Baalen, N. Perrin and L. Lehmann for helpful comments. The manuscript benefited greatly from the comments of two referees and the encouragoffing input by the Associate Editor. This work was supported by grants from the Adaptive Dynamics Network at the International Institute for Applied System Analysis (Laxenburg, Austria), the French Ministry of Research and Education, and the European Science Foundation (Theoretical Biology of Adaptation Programme, Travel Grant). Collaboration on this study has also been fostered by the European Research Training Network ModLife (Modern Life-History Theory and its Application to the Management of Natural Resources), supported by the Fifth Framework Programme of the European Community (Contract Number HPRN-CT-2000-00051). 


\title{
The Adaptive Dynamics of Altruism in Spatially Heterogeneous Populations
}

\author{
Jean-Francois Le Galliard \\ Régis Ferrière \\ Ulf Dieckmann
}

Altruism is a cooperative behavior by which a donor individual increases a recipient's fitness at the cost of its own fitness. Major progress in the study of the evolution of altruism has been made over the last decade on both theoretical and empirical sides. On the theoretical side, models have gained a significant dose of realism from the explicit inclusion of spatial factors and the consideration of conditional behavior (Nowak and May 1992; Ferrière and Michod 1995; Roberts and Sherrat 1998). On the empirical side, ecological and genetic determinants of altruistic behavior have started to be identified, and physiological costs and benefits have been measured (Crespi 1996; Bourke 1997; Cockburn 1998; Heinsohn and Legge 1999). However, the merging between theory and facts has led to conflicting interpretations of processes and patterns in the evolution of altruism.

Two main processes have been put forward to explain the evolution and maintenance of altruism: kin selection (Hamilton 1964) and reciprocity (Trivers 1971; Axelrod and Hamilton 1981). Kin selection initially met with great success in explaining empirical observations (for example in social insects). However, recent theoretical developments, based on spatially implicit models, have pointed out a critical issue in this framework: the deleterious effects of kin competition should cancel out the indirect benefits of an altruistic behavior, thereby preventing the evolution of altruism (Taylor 1992a, 1992b; Wilson et al. 1992; Queller 1992, 1994). In contrast, game-theoretic spatial models involving conditional reciprocity have shown that reciprocal altruism evolves readily in spatially heterogeneous populations (Nakamaru et al. 1997, 1998). These theoretical findings are altogether in sharp contrast with empirical advances. On the one hand, unequivocal evidence is lacking for the expectedly widespread occurrence of reciprocal altruism, and reciprocity involves already fairly elaborate behavioral mechanisms (e.g., memory of past interactions) that are unlikely to be relevant to our understanding of the evolution of primitive forms of altruism (Pusey and Packer 1997). On the other hand, kin selection is still regarded as essential to explain the transition from selfish to cooperative units at all levels of biological organization (Maynard-Smith and Szathmary 1995), and many empirical examples of the specific transition from solitary to social life in animals seem indeed to fall under the scope of kin selection (Bourke 1997; Emlen 1997). 
Widely different patterns of altruistic behavior have been described in a large array of taxa spread across bacteria, slime moulds, arachnids, insects and vertebrates. Levels of altruism, as described by the qualitative and quantitative natures of the investment by donors and benefit to recipients, have been found to vary between different species (Edwards and Naeem 1993; Crespi 1996) or within the same lineage across evolutionary time (Jarvis et al. 1994; Wcislo and Danforth 1997), between different populations within the same species (Spinks et al. 2000) and between groups of individuals within the same population (Cockburn 1998; Velicer et al. 2000; Strassmann et al. 2000). Most models have aimed at understanding how altruism can evolve in a selfish world, and how altruists can persist in the face of cheaters that reap the benefits of altruism while providing less or no help. Yet little theory is currently available to probe the adaptive significance of such variation in patterns of altruism and to identify physiological, ecological and genetic determinants.

This study offers a theoretical framework to address these tensions. We consider a population of asexual organisms that live in a spatially homogeneous, temporally constant habitat where competition and cooperation take place between kin and non-kin neighbors (van Baalen and Rand 1998). First, we address the robustness of previous investigations that questioned the role of kin selection for the evolution of altruism. To this end, we relax two of their critical assumptions. A dose of movement may help export the local benefits of altruism. Thus, we expect the conclusion that kin competition cancels kin cooperation to be sensitive to the inclusion and intensity of individual mobility (Queller 1992). We therefore relax the "pure viscosity" hypothesis according to which individuals (except offspring) are sessile (Hamilton 1964; Taylor 1992a,b). In our model, offspring are born locally, but in contrast with most viscous population models, individuals move during their lifetime (van Baalen and Rand 1998). Also, most kin selection models assume that the population is saturated and constantly maintained at carrying capacity. This lack of environmental "elasticity" might prevent the spread of altruism (Queller 1992, Mitteldorf and Wilson 2000). To overcome this restriction we assume that population regulation arises locally from the limited empty space being available for offspring. In our model the habitat is not saturated, because occupied sites coexist with empty sites generated by demographic stochasticity (Ferrière and Le Galliard 2001).

Second, we want to understand adaptive variation in altruism from basic physiological, ecological and genetic properties that could be documented in natural populations. This is achieved by assuming that altruism is not an all-or-nothing behavior and is better modeled as a quantitative trait that measures the amount of time, energy or resources invested in the altruistic function (Doebeli and Knowlton 1998; Roberts and Sherrat 1998; Koella 2000). At the physiological level, populations or species may differ according to the pattern of energy allocation to altruism versus other costly functions (Heinsohn and Legge 1999). We therefore assume that the physiological cost of altruism relates quantitatively to the actual altruistic investment. However, in contrast with previous models (Doebeli and Knowlton 1998; Roberts and Sherrat 1998), we envision three alternative costs patterns: a decelerating, a linear and an accelerating dependence of costs on investment in altruism. At the level of ecology, populations or 
species may also differ with respect to interaction structure. Two determinants of this structure are habitat connectedness and individual mobility (Ferrière and Michod 1995, 1996; Nakamaru et al. 1997; Frank 1998). Introducing specific parameters for these two factors allows us to investigate their effect on the adaptive evolution of altruism. At the level of the genetic processes, mutation rates and mutational effects determine the population phenotypic diversity upon which selection operates. Our study addresses to what extent variations in these basic genetic features can contribute to variations in adaptive patterns of altruism.

From a methodological point of view, we develop a model of population dynamics based on spatial correlation equations (Matsuda et al. 1992; van Baalen and Rand 1998; Rand 1999; Ferrière and Le Galliard 2001) to study the evolutionary dynamics of altruism in the framework of adaptive dynamics theory (Metz et al. 1996; Dieckmann and Law 1996). The central notion is that selective pressures acting on mutant phenotypes are generated by the background population dynamics of resident phenotypes. After identifying selective pressures and incorporating them in a deterministic model of, we provide a classification of adaptive patterns of altruism according to the shape of physiological costs, the levels of individual mobility and adaptive dynamics the degree of habitat connectivity. The stability of the evolutionary endpoints and the effect of large mutations are investigated to gain insight into how variation in the mutation process may determine the adaptive outcome. Finally, the robustness of the salient conclusions drawn from our analytical study is tested against stochastic, individual-based simulations.

\section{Model assumptions}

We consider a population of haploid individuals that inhabit a network of homogeneous sites, modeled as an infinite lattice (Appendix 1). Each site may be empty, or occupied by one individual. Each site is randomly connected to a set of sites that defines a neighborhood (Appendix 1). We assume that every site is connected to the same number of sites, $n$. Thus, the neighborhood size $n$ provides a measure of the habitat's connectedness.

Mobility and interaction are defined locally, at the neighborhood scale. During any small time interval, an individual may move to an empty site within its neighborhood, reproduce by putting an offspring into an empty neighboring site, or die. The per capita mobility rate $m$ and death rate $d$ are unaffected by local interactions. Mobility is assumed to be costly to the individual, with a permanent negative effect on the individual's birth rate. This is expected in organisms where a stronger ability to move, resulting from specific structures (e.g., gliding flagella or muscles), imposes a developmental or maintenance cost. For example, the dispersive morph in the naked mole rat diverts more energy into growth and fat storage to compensate for the risks of moving in inhospitable habitats (O'Riain et al. 1996). The cost of mobility is assumed to impact linearly on the intrinsic birth rate such that the net per capita birth rate (in the absence of interaction) is given by $b(m)=b-v m$, where $b$ measures the intrinsic per capita birth rate in sessile organisms that do not invest energy into mobility, and $v$ measures the sensitivity of the cost to mobility. 


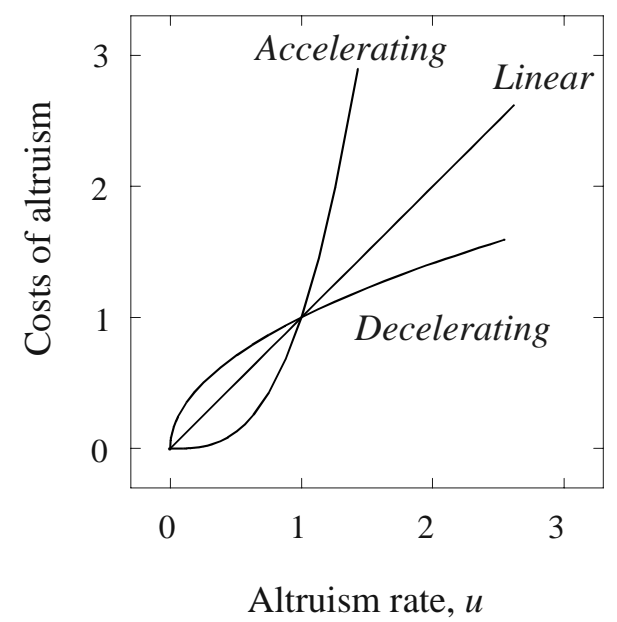

F igu $\mathrm{re} 1$. Costs of altruism as a function of the individual investment in altruism $(u)$. This function is given by $C(u)=\kappa u^{\gamma}$ (with $\kappa=1$ in this display). Decelerating costs, $\gamma<1$ : the cost increases with the rate of altruism first steeply and then more slowly. This pattern makes the origin of altruism from selfishness harder. Accelerating costs, $\gamma>1$ : the cost increases with the rate of altruism first slowly and then steeply. This pattern turns out to influence the long-term adaptive level of altruism. Linear costs, $\gamma=1$ : the rate of increase of the cost is independent of the level of altruism.

We assume that two types of local density-dependent factors affect movement and reproduction (Appendix 1). First, both movement and reproduction are conditional on the availability of empty sites within the neighborhood. Thus, local crowding negatively affects the rates of mobility and birth. Second, reproduction is enhanced by altruistic interactions with neighboring individuals, which induces a positive effect of local crowding. Here we assume that an altruistic interaction improves the quality of neighboring sites. This may involve storage of resources, habitat engineering, or signaling. The altruistic phenotype is defined by the per capita rate of investment $u$ into the altruistic function. The altruistic behavior is directed evenly toward all neighboring sites, regardless of the presence or phenotypes of neighbors. In effect, every neighbor of a focal individual that invests at rate $u$ into altruism receives a birth rate increment equal to $u / n$ (Wilson et al. 1992). Therefore, altruism is only effective in practice provided some recipients are present in the neighborhood of the donor and some space is available in the neighborhood of the recipient for its offspring. We use the terms selfishness to describe a phenotype that does not invest in altruism $(u=0)$ and quasiselfishness to refer to a phenotype that hardly invests in altruism $(u \approx 0)$.

We further assume that altruism involves a physiological cost on the donor's reproduction, and we distinguish three patterns of dependence of costs on the investment in altruism: accelerating, linear, and decelerating (Fig. 1). With accelerating costs, the increase of the cost resulting from an increased altruistic investment becomes disproportionately larger as the initial investment increases. For example, in the cooperative bird Corcorax melanorhamphos physiological costs are detected only among individuals that invest strongly in altruism (Heinsohn and Cockburn 1994). 
Conversely, a decelerating pattern yields a disproportionate increase of costs at lower investment. This would apply to organisms in which the initiation of altruism from a selfish state would be very costly. In the limiting case of a linear pattern, the cost sensitivity is independent of the level of investment. The physiological cost of altruism $C(u)$ is modeled as a simple, allometric function that encapsulates the three patterns of decelerating, linear, and accelerating costs: $C(u)=\kappa u^{\gamma}$, where $\kappa$ scales the sensitivity of the cost to the investment $(\kappa>0)$, and $\gamma$ determines whether costs are accelerating $(\gamma>1)$, linear $(\gamma=1)$ or decelerating $(\gamma<1)$.

Mutations cause the altruistic phenotypes of offspring to differ from those of their parents. Mutations occur with a fixed probability per birth event, denoted by $k$. The mutant phenotype is obtained by adding the mutation effect to the progenitor phenotype. Mutation effects are drawn randomly from a normal probability distribution, with zero mean and a mutational variance $\sigma^{2}$. The resulting polymorphic, stochastic process can be simulated on a finite lattice (Appendix 1). Table 1 summarizes the used notation.

\section{Spatial population dynamics of residents}

Selective pressures acting on a mutant phenotype result from the interaction between the initially scarce mutant population and the resident background population. The finite size of the interaction neighborhood and the finite range and rate of dispersal and fecundity cause spatial fluctuations and spatial correlations to develop in population density (Dieckmann and Law 2000). Thus, spatial population heterogeneity develops in a spatially homogeneous habitat. We use the framework of correlation equations to derive an analytical model describing the spatial dynamics of such a background population (Appendix 2). Assuming that mutation occurs rarely, the background population may be considered as monomorphic (Dieckmann and Law 1996; Metz et al. 1996). In this section we apply the polymorphic, ecological model to the specific case of a monomorphic population. This will provide the basic ingredients needed in the next section to model the dynamics of a mutant phenotype situation in this resident population.

Let us consider a single phenotype $x$ which invests in altruism at rate $u_{x}$. The temporal dynamics of a population of $x$ can be described by tracking over time $t$ the frequency $p_{x}(t)$ of occupied sites. These dynamics depend on the neighborhood composition, described by the local frequencies $q_{i \mid x}$, i.e. the probabilities that an occupied site is neighbored by at least one site in the state $i$ (Matsuda et al. 1992). The frequency $p_{x}$ i obeys the ordinary differential equation

$$
\frac{d p_{x}(t)}{d t}=\left[\left(b(m)+(1-\phi) \cdot u_{x} \cdot q_{x \mid x}(t)-C\left(u_{x}\right)\right) \cdot q_{0 \mid x}(t)-d\right] \cdot p_{x}(t),
$$


I a ble 1. Notations used in this paper

\begin{tabular}{|ll|}
\hline Model parameters \\
$n$ & Neighborhood size $(\phi=1 / n)$ \\
$b$ & Intrinsic birth rate \\
$d$ & Intrinsic death rate \\
$m$ & Intrinsic mobility rate \\
$u_{i}$ & Intrinsic investment rate in altruism of a phenotype $i$ (adaptive trait) \\
$C\left(u_{i}\right)$ & Cost of altruism, impacting the birth rate of a phenotype $i$ \\
$\kappa$ & Cost sensitivity with respect to the level of investment in altruism \\
$\gamma$ & Cost acceleration with respect to the level of investment in altruism \\
$k$ & Mutation probability per birth event \\
$\sigma^{2}$ & Mutational step variance \\
Model variables \\
$p_{i}$ & Global frequency of sites $i$ \\
$q_{i \mid j}$ & Local frequency of sites $i$ neighboring a $j$ site \\
$\bar{p}_{x}$ & Equilibrium global frequency of a resident $x$ \\
$\bar{q}_{i \mid x}$ & Equilibrium local frequency of sites $i$ neighboring a resident $x$ \\
$\tilde{q}_{i \mid y}$ & Pseudo-equilibrium frequency of sites $i$ neighboring a rare mutant $y$ \\
\hline
\end{tabular}

involving the local frequencies $q_{x \mid x}(t)$ and $q_{0 \mid x}(t)$ of occupied and empty sites next to an occupied site at time $t$, and $\phi=1 / n$. A closed system of correlation equations for the dynamics of local frequencies is constructed in Appendix 2 by making use of the standard pair approximation (Matsuda et al. 1992; Rand 1999; Iwasa 2000; van Baalen 2000).

At equilibrium, the spatial structure of a monomorphic population depends on the mobility rate and the altruistic investment (Appendix 3). The spatial structure is characterized by some degree of aggregation (Fig. 2). The spatial structure vanishes at high mobility rates, and for large birth rates, because birth is associated with offspring dispersal. More aggregation is found in organisms with low mobility, and also in organisms with very high mobility that consequently incur a severe reduction of their birth rate (due to the cost of mobility). The relationship between altruism and aggregation depends on the pattern of cost. In species with linear and decelerating costs, strongest aggregation is observed at low altruistic investment (Figs. 2B to D). In species with accelerating costs, strongest aggregation is observed in organisms with low altruism, or with high altruism, when the birth rate is drastically reduced by the cost of altruism (Fig. 2F). 


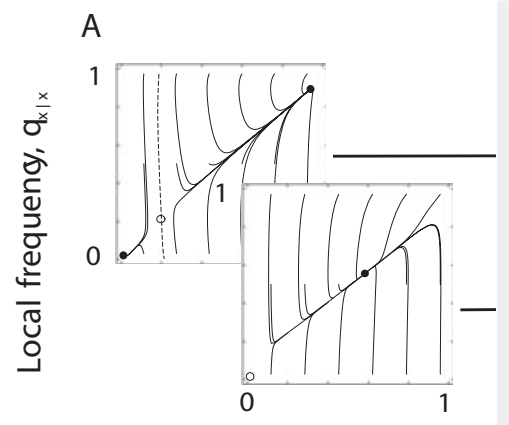

Global frequency $p_{x}$

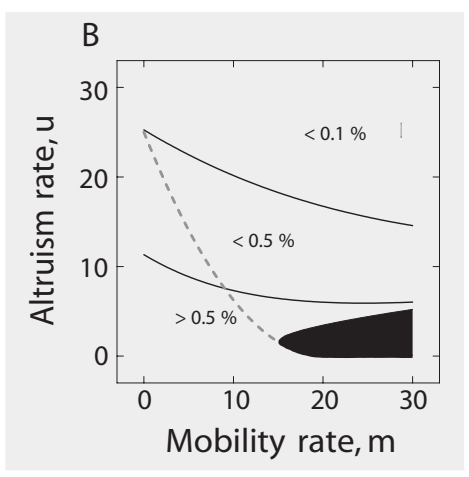

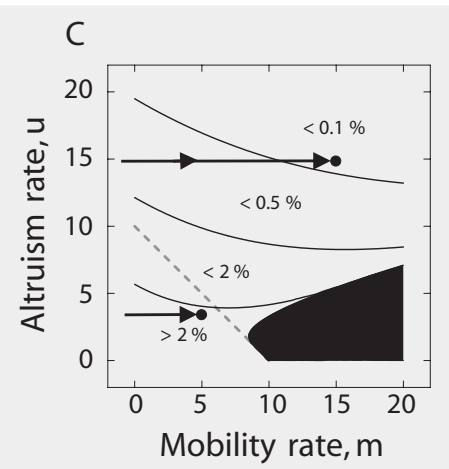

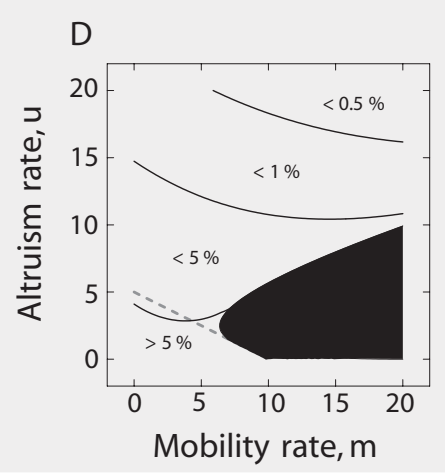

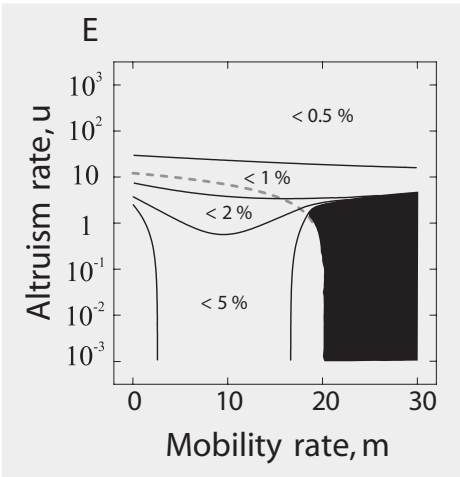

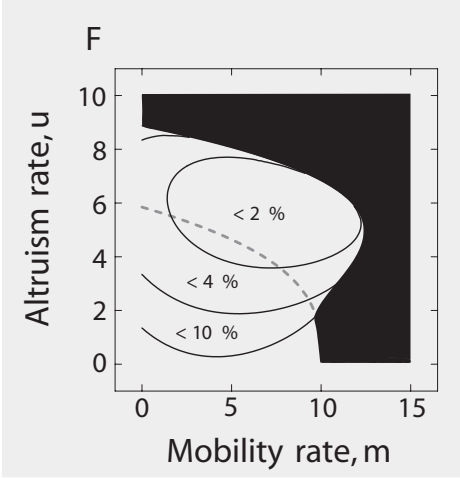

F ig u p e 2. Monomorphic population dynamics. A, Population trajectories of global and local frequencies, as predicted by Equations (A4) and (A10). Starting from any initially rare state, the local frequency converges fast toward a one-dimension manifold along which most of the global frequency dynamics take place. The upper panel shows a case of obligate altruism $(m=15$, $u_{x}=20$ ): a positive equilibrium and the extinction equilibrium are both locally stable (filled circles) and coexist with a saddle point (open circle). The dotted curve separates the basins of attraction of the two stable equilibria. The lower panel shows a case of facultative altruism ( $m=5, u_{x}=3$ ): there is a globally stable equilibrium (filled circle); the extinction equilibrium (open circle) is unstable. Parameters: $\gamma=1, \kappa=0.1$, and $v=0.1$. B-F, Parameter regions of facultative altruism (below dashed curve), obligate altruism (above dashed curve) and population extinction (black area). Black curves are contours of the relative percent of deviation of spatial structure from mean-field equilibrium. B, Species with decelerating costs $(\gamma=0.5$, $\kappa=0.2$, and $v=0.05)$. C, Species with weak linear costs (parameter values as in A). D, Species with strong linear costs $(\gamma=1, \kappa=0.2$, and $v=0.1)$. E, Species with slowly accelerating costs $(\gamma=1.2, \kappa=0.05$, and $\nu=0.05)$; extinction also occurs at higher values of altruistic investment (not shown). F, Species with rapidly accelerating costs ( $\gamma=3, \kappa=0.005$, and $v=0.1$ ). Life-history and connectedness parameters are $b=2, d=1$ and $n=4$, here as well as in all other figures.

For some parameter combinations, extinction is the only stable population equilibrium (Fig. 2). Extinction results from the total cost of mobility and altruism not being compensated. High mobility causes extinction because it implies a large direct cost that depresses the intrinsic birth rate $b(m)$, along with the reduction of the indirect benefits of altruism due to the loss of local aggregation. Species with accelerating costs can also undergo extinction at high investment in altruism (Fig. 2F). In all other cases, 
the altruistic population is viable and two types of population dynamics can be distinguished (Fig. 2). Borrowing terminology from the field of mutualism studies, we call the corresponding phenotypes facultative versus obligate. Thus, altruism is said to be facultative when the population growth rate in the limit of very low population density, $b(m)-d-C\left(u_{x}\right)$, is positive. The population is then characterized by a single, globally stable, and positive equilibrium $\left(\bar{p}_{x}, \bar{q}_{x \mid x}\right)$ (Fig. 2A). Altruism is said to be obligate when the limit growth rate is negative and the population dynamics thus show bistability. In the latter case, the positive equilibrium is locally stable and coexists with the extinction equilibrium, which is also locally stable. A population of obligate altruists may attain the viable equilibrium state only if its initial density lies above a critical threshold (Fig. 2A). This phenotypic state is therefore associated with low colonization ability and an elevated risk of extinction, since a viable population can neither be established from an initially low density nor be maintained below a critical density threshold.

\section{Spatial invasion fitness of mutants}

The invasion fitness of a mutant is defined by its per capita growth rate while being rare in a resident population at ecological equilibrium (Metz et al. 1992). In the present section, we analyze the growth of such a small mutant population in a resident population as described in the previous section (Appendix 4).

The invasion dynamics of a rare phenotype involves three phases (van Baalen 2000; Fig. 3). In a first, short phase, the small mutant population locally spreads from a single mutant individual up to the point where the mutant population attains a pseudoequilibrium correlation structure. The build-up of this structure is highly stochastic but occurs with certainty on a finite time scale (Matsuda et al. 1992). Indeed, the cost of altruism dooms any single altruistic mutant in an established population of selfish individuals. Drift is first needed to drive the mutant population to its pseudo-equilibrium spatial structure. Also, the initial spread of the mutant depends on the local spatial structure of the resident population. For example, an altruistic mutant that arises in a neighborhood where selfish residents are more frequent than expected on average will face an increased risk of extinction. Denoting the mutant by $y$, we use the pseudoequilibrium local frequencies $\tilde{q}_{0 \mid y}, \tilde{q}_{x \mid y}$ and $\tilde{q}_{y \mid y}$ of (respectively) empty, resident and mutant sites around a focal mutant site to describe this transient structure. These statistics are calculated in Appendix 5. Conditional on non-extinction during this first phase, the mutant dynamics then enter the second phase during which the mutant population expands or contracts while its population keeps its pseudo-equilibrium structure and the resident population remains close to its own equilibrium (Fig. 3). Spatial invasion fitness can be defined as the mutant population growth rate during this second phase (van Baalen and Rand 1998). A positive fitness implies that the invasion process enters a third phase during which the mutant phenotype displaces the resident (Fig. 3), while a negative fitness implies mutant population extinction. 




F ig u re 3. Successful invasion of an initially rare, altruistic mutant $\left(u_{y}=1\right)$ into a selfish resident population at equilibrium. Dynamics of the resident global frequency $\left(p_{x}\right)$ and of the mutant global $\left(p_{y}\right)$ and local frequency $\left(q_{y \mid y}\right)$, as predicted by the deterministic system of correlation equations (A13). The three phases of invasion apparent in the dynamics of $q_{y \mid y}$ are discussed in the text. Parameter values: $\gamma=2$ (accelerating costs), $\kappa=0.1, v=0.1$, and $m=0$.

The spatial invasion fitness $s_{x}(y)$ can be expressed as a function of the pseudoequilibrium statistics of the mutant population. Combining the population growth rate (1) with the expression of the mutant pseudo-equilibrium local frequencies, $s_{x}(y)$ is given by

$$
s_{x}(y)=\left[\left(b(m)-C\left(u_{y}\right)\right) \tilde{q}_{0 \mid y}-d\right]+(n-1) \phi u_{x} \tilde{q}_{0 \mid y} \tilde{q}_{x \mid y}+(n-1) \phi u_{y} \tilde{q}_{0 \mid y} \tilde{q}_{y \mid y} .
$$

Notice that the benefit of altruism (second and third terms) is measured conditionally on the presence of at least one empty site for breeding and depends upon the amount of help received from $n-1$ (not $n$ ) neighboring sites and the local frequency of empty sites $\tilde{q}_{0 \mid y}$.

This expression bears an interesting relationship to the notion of direct or neighbormodulated fitness of additive behavioral effects (Frank 1998). Direct fitness is derived by summing the effects on a focal individual's fitness of all phenotypes present in the neighborhood (including the focal individual itself). Likewise, the spatial invasion fitness of a focal mutant is obtained by adding to the mutant neighbor-independent fitness (first term) the effects of a resident neighbor (second term) and that of a mutant neighbor (third term), weighed by the probabilities of occurrence of such neighbors.

\section{Selective pressures}

We now derive a simplified version of the spatial invasion fitness to analyze the selective pressures acting on the altruistic trait under small mutational steps. This will be the basis for studying the evolutionary dynamics of altruism. By using the fact that the resident's fitness in its own environment is always zero, $s_{x}(x)=0$, the selection derivative can be derived from a first-order approximation of the spatial invasion fitness, and equals 


$$
\left.\frac{\partial s_{x}(y)}{\partial y}\right|_{y=x}=\bar{q}_{0 \mid x}\left((1-\phi) \bar{q}_{y \mid y}-a\left((1-\phi) u_{x}-\frac{d}{\bar{q}_{0 \mid x}^{2}}\right)-\left.\frac{\partial C\left(u_{y}\right)}{\partial u_{y}}\right|_{y=x}\right),
$$

where $a$ measures the gain (or loss) of open space in a mutant's pseudo-equilibrium neighborhood relative to the resident's at equilibrium (see Appendix 5 for details). This expression exhibits three selective pressures driving the evolution of altruism. The first term on the right-hand side of Equation (3) quantifies the pressure for increased investment in altruism. The second term measures the pressure for opening free space in an individual's neighborhood. The last term measures the pressure for reducing the physiological cost of investing in altruism.

Extensive computations suggested that the pressure for opening space, albeit not vanishing, is negligible compared to the two other selective components (Fig. 4). This implies that $\bar{q}_{0 \mid x} \approx \tilde{q}_{0 \mid y}$, hence $a \approx 0$ in the Equation (3) (see Appendix 5). This also implies that as long as the mutant phenotype stays rare, the resident correlation structure is redistributed over the pairings of mutants with their own type and the resident type: $\bar{q}_{0 \mid x}=\tilde{q}_{y \mid y}+\tilde{q}_{x \mid y}$. Thus, the mutant is less aggregated than expected when common, and therefore rare mutants are less likely to interact among themselves during the initial phases of invasion (Fig. 3). Globally, the evolution of altruism is not limited by competition for empty sites within the invasion structure, and the condition for an adaptive increase in altruism, $\partial s_{x}(y) / \partial y>0$, is equivalent to

$$
(1-\phi) \tilde{q}_{y \mid y}>\left.\frac{\partial C\left(u_{y}\right)}{\partial u_{y}}\right|_{y=x}
$$

This condition is a spatial form of Hamilton's rule (see also Ferrière and Michod 1995, 1996; Frank 1998; van Baalen and Rand 1998). The right-hand side is the marginal cost of altruism. The left-hand side measures the marginal benefit of altruism, weighed by the average frequency $\tilde{q}_{y \mid y}$ of recipient neighbors that are phenotypically identical to the focal mutant individual. For a haploid mutant population descended from a single mutation event, the identity in phenotype is equivalent to the identity by descent, and $\tilde{q}_{y \mid y}$ provides a measure of relatedness (Day and Taylor 1998).

The mutant relatedness can be expressed as a function of the resident population structure (Appendix 5), hence in terms of the basic demographic, mobility and lattice parameters, according to the following equality

$$
\tilde{q}_{y \mid y}=\frac{d \phi}{d+(1-\phi) m \bar{q}_{0 \mid x}} .
$$

Thus relatedness is higher in a population with lower $\bar{q}_{0 \mid x}$, which promotes the invasion of even more altruistic phenotypes. Insofar as the local frequency $\bar{q}_{0 \mid x}$ correlates negatively with the investment $u_{x}$, this relation establishes a positive ecological feedback on the evolution of altruism: a negative effect of altruism investment on the local frequency $\bar{q}_{0 \mid x}$ increases the relatedness of mutants, thereby enhancing the selective pressure for increased altruism. Also, Equation (5) shows that there is a direct negative effect of mobility on mutant relatedness, and an indirect effect through $\bar{q}_{0 \mid x}$. 

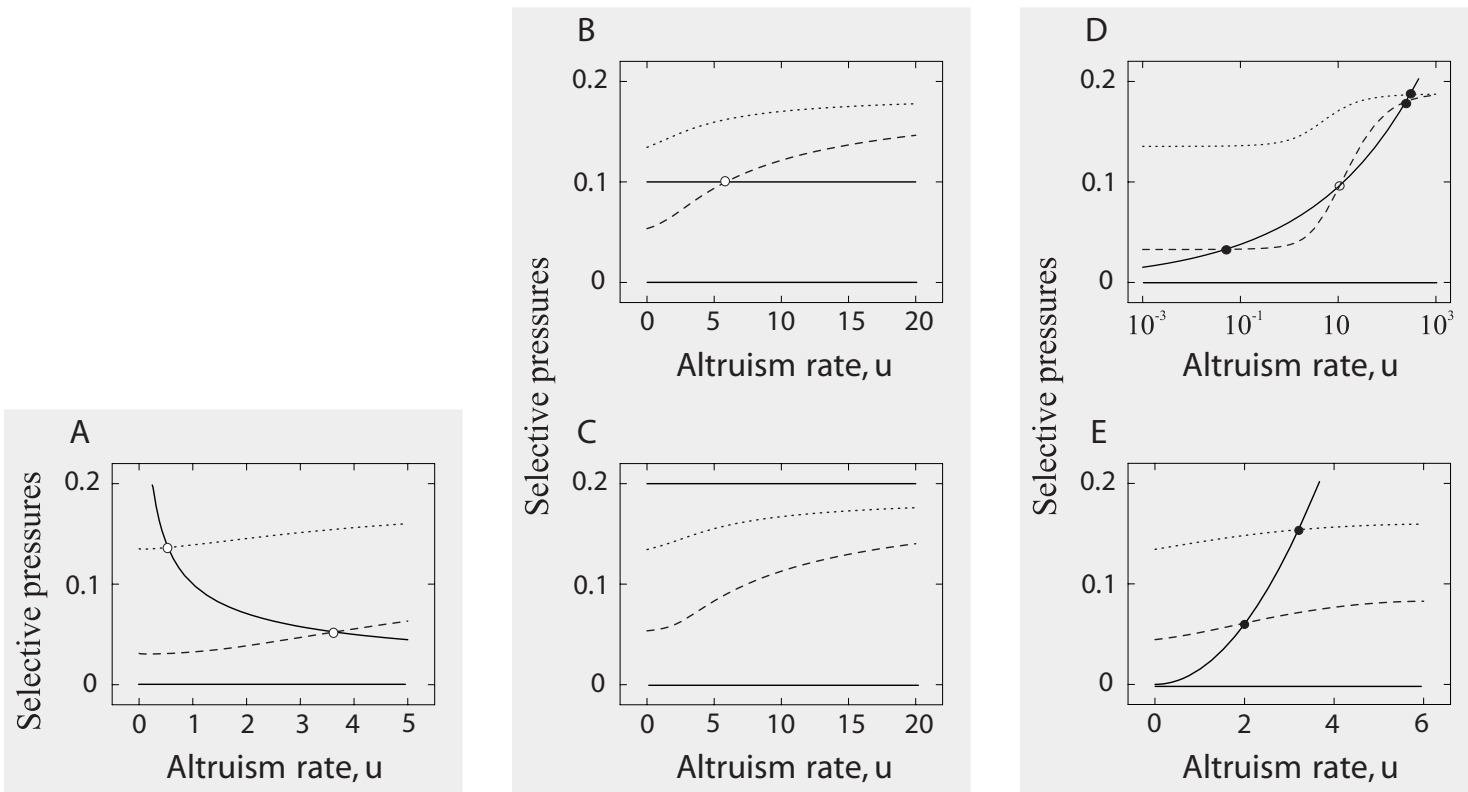

F ig u r e 4 : Selective pressures acting on altruism under small mutational steps calculated by following the recipe described in Appendix 5. Broken curves: positive pressure for increasing altruism, $(1-\phi) \bar{q}_{y y}$, evaluated at low ( $m=1$, dotted curves) and high mobility ( $m=10$, dashed curves). Continuous curve: negative pressure for reducing the cost of altruism. Lower continuous line: negative, negligible selective pressure for decreasing local competition. Within the range of altruism where a broken curve is below (above) the continuous curve, selection favors the increase (decrease) of altruism. Circles indicate singular points where selective pressures exactly balance each other. Filled circles: attracting evolutionary singularities. Open circles: repelling evolutionary singularities. Parameter values in A to $\mathrm{E}$ are the same, respectively, as in Fig. 2B to F.

Both effects add up to decrease mutant relatedness and weaken the selective pressure that favors altruism. Finally, increasing the neighborhood size $n$ decreases $\tilde{q}_{y \mid y}$ : a larger neighborhood size hampers the evolution of altruism.

\section{Canonical equation of adaptive dynamics}

We use the results of the previous section to develop a deterministic model of adaptive dynamics under small mutations. This allows us to identify general patterns in the adaptive dynamics of altruism, to characterize the evolutionary endpoints and to study transient evolutionary dynamics. In a large population where mutations are rare and mutational steps are small, the stochastic mutation-selection process can be approximated by a deterministic process whose trajectories are the solution of the socalled canonical equation of adaptive dynamics (Dieckmann and Law 1996):

$$
\frac{d u_{x}}{d t}=\left.\left[k \cdot \frac{\sigma^{2}}{2} \cdot \bar{p}_{x}\right] \cdot \frac{\partial s_{x}(y)}{\partial y}\right|_{y=x} .
$$

The bracketed term captures the effect of mutations, involving the mutation probability $k$, the mutation step variance $\sigma^{2}$, and the equilibrium population frequency $\bar{p}_{x}$ of a monomorphic population of phenotype $u_{x}$. The local direction of phenotypic change is 
given by the selection derivative (Marrow et al. 1992) that we approximate according to Equation (4) by

$$
\left.\frac{\partial s_{x}(y)}{\partial y}\right|_{y=x}=\bar{q}_{o: x}\left[(1-\phi) \bar{q}_{y \mid y}-\left.\frac{\partial C(u)}{\partial u}\right|_{u=u_{x}}\right] .
$$

The resting points that satisfy $d u_{x} / d t=0$ are called evolutionary singularities and correspond to phenotypic states where the selection derivative vanishes (Marrow et al. 1992). Thus, at an evolutionary singularity, the marginal cost of altruism balances exactly the marginal benefit weighted by mutant relatedness. A singularity $u^{*}$ can be locally evolutionarily attractive ("convergence stable"), or acts as an evolutionarily repellor.

\section{Classification of Adaptive Dynamics}

We develop a classification of the adaptive dynamics of altruism depending on the cost pattern specified by parameters $\kappa$ and $\gamma$. To this end, we perform a numerical bifurcation analysis of the evolutionary singularities generated by (6) and (7) with respect to the mobility rate. We obtain five generic bifurcation diagrams as parameters $\kappa$ and $\gamma$ are varied (Fig. 5A).

For a decelerating cost of altruism, there is a single positive singularity that is unstable for any mobility rate (Fig. 5B). For linear costs, two cases can be distinguished. Either the selfish state undergoes a transcritical bifurcation as mobility increases, turning from unstable to stable and then coexisting with an unstable positive singularity. This is characteristic of "weak linear costs" (Fig. 5C). Alternatively the selfish singularity remains stable irrespective of the mobility rate, which characterizes "strong linear costs" (Fig. 5D). For accelerating costs, there are also two distinct patterns. For low values of $\kappa$ and $\gamma$, there is a range of intermediate mobility rates over which the adaptive dynamics of altruism possess one unstable and two stable singularities. As mobility decreases, the lower stable equilibrium and the unstable one collide, leaving the upper stable singularity alone. Such combinations of $\gamma$ and $\kappa$ values define "slowly accelerating costs" (Fig. 5E). For higher values of $\kappa$ and $\gamma$, characterizing "rapidly accelerating costs", there is a single stable positive equilibrium for every mobility rate (Fig. 5F).

Generically, natural selection favors altruism in species characterized by a large intrinsic birth rate $b$ and a small death rate $d$. Also, altruism is selected against in species characterized by a large mobility rate. Selection against the altruistic trait may even lead to the "evolutionary suicide" of the population, if the adaptive dynamics start from an intermediate level of altruism and a high level of mobility (Figs. 5C to F). 

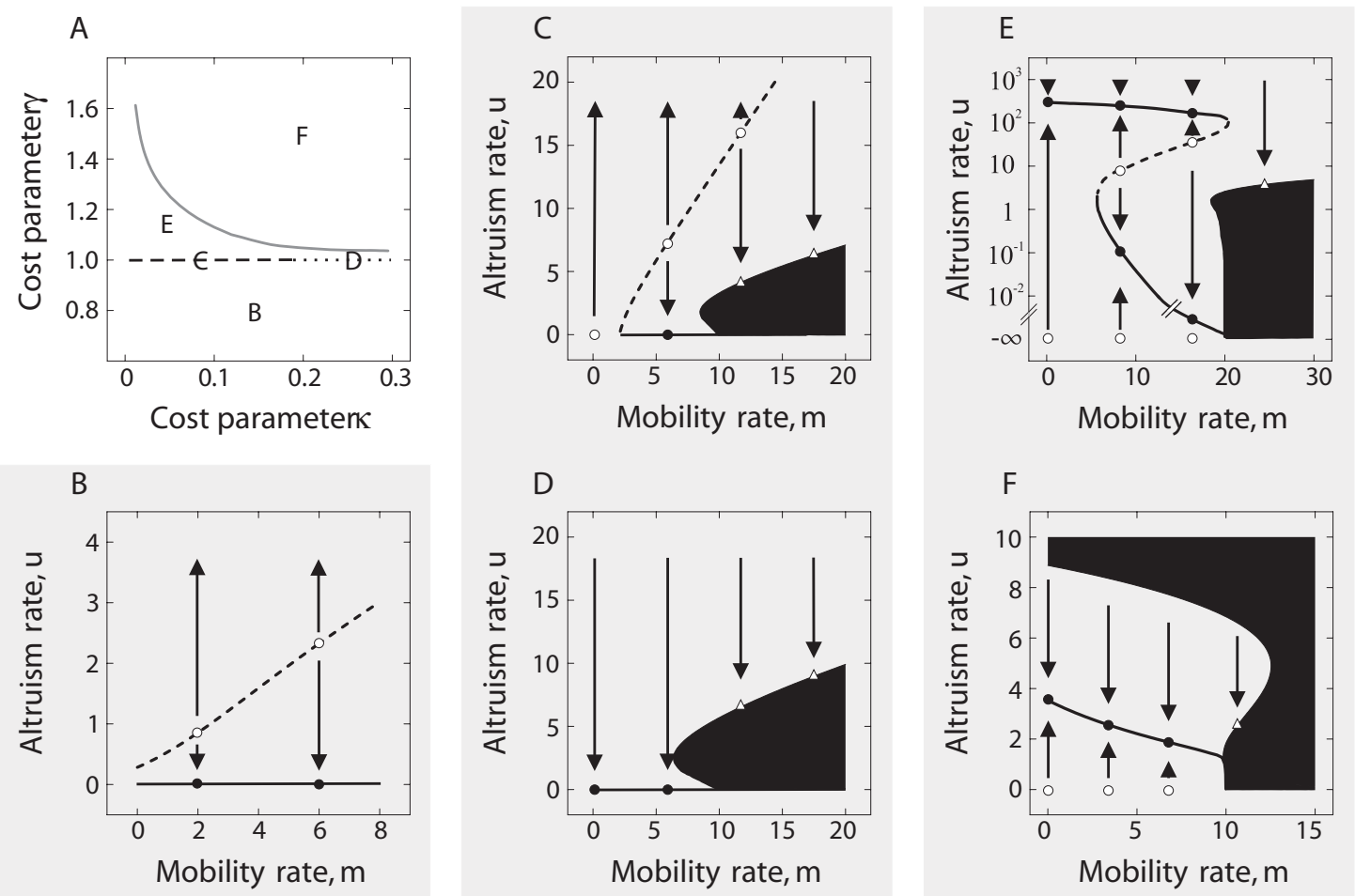

F ig u r e 3 . Adaptive dynamics of altruism. A, Classification of adaptive dynamics according to cost parameters $\gamma$ and $\kappa$. Lettering refers to panels B to $\mathrm{F}$ showing bifurcation diagrams of the evolutionary singularities with respect to the mobility rate. In B-F, plain black curves are sets of convergence stable (attracting) singularities; dashed black curves are sets of convergence unstable (repelling) singularities. Population extinction occurs in black regions. Arrows indicate the direction of selective pressures at particular values of the mobility rate. Filled circles: attractive evolutionary singularity; open circles: repelling evolutionary singularity; triangles: evolutionary self-extinction. B, Species with decelerating costs. Inner singularities are repelling, resulting in bistable adaptive dynamics. $\mathrm{C}$, Species with weak linear costs. Below a mobility threshold, altruism invades a purely selfish population and increases monotonically; above the threshold, the adaptive dynamics are bistable. D, Species with strong linear costs. Pure selfishness is globally attractive. E, Species with slowly accelerating costs. High altruistic investments are selected at low mobility. At higher mobility, an unstable singularity separates the basins of attraction of two locally attracting singularities that differ dramatically in their level of altruism (high altruistic investment versus quasiselfishness). F, Species with rapidly accelerating costs. The adaptive dynamics typically converge to a globally stable singularity. In all cases (not shown in B), the adaptive process can hit a region of extinction when the population originates from an ancestral state characterized by high mobility and intermediate or high altruism. Values of parameters $\kappa$ and $\gamma$ in B to $\mathrm{F}$ are the same, respectively, as in Fig. 2B to F. In all panels $v=0.1$.

Although highly mobile organisms could persist on the ecological timescale provided that they behave sufficiently altruistically, the adaptive process would drive their altruistic investment down to the point where the population becomes non-viable. 
Increasing the size of the neighborhood selects strongly against altruism. The whole patterns are not sensitive to variations in the mobility cost parameter $v$.

\section{Decelerating Costs}

With decelerating costs, there is a single, repelling evolutionary singularity for any mobility rate, and adaptive dynamics exhibit bistability (Fig. 5B). At the evolutionary singularity, the positive selective pressure on altruism resulting from mutant relatedness and the negative pressure exerted by the physiological cost balance exactly. The evolutionary singularity increases with the mobility rate, which is due to the effect of increased mobility on mutant relatedness as described by Equation (5).

Since the cost pattern is decelerating, a slight increase of altruism within the range below the singularity is counter-selected since it induces a cost that is disproportionately larger than the gain. As a result, the adaptive dynamics ought to converge to selfishness (Fig. 4A). If the ancestral population state is sufficiently altruistic, the adaptive process will result in ever-increasing altruism. This is because, with decelerating costs, the cost of altruism increases more slowly than the benefits of altruism resulting from increased relatedness (Fig. 4A). In real systems, the adaptive increase of altruism should be limited by physiological or functional constraints, and the evolutionary process is expected to halt at such a limiting trait value.

\section{Linear Costs}

The adaptive dynamics of altruism in species with linear costs can be classified in two categories according to the cost parameter $\kappa$ (Fig. 5A). For species with low $\kappa$, costs are said to be "weak linear" and the adaptive dynamics depend on the mobility rate (Fig. $5 \mathrm{C})$. For low mobility, the selfish state is invadable by altruism and the adaptive process leads to the maximum physiologically feasible investment in altruism. Above a threshold on mobility, there exists a positive, repelling singularity and the adaptive process behaves as in the case of decelerating costs. If the initial investment in altruism lies below the singularity, the marginal benefit is too low to compensate for the marginal cost, and decreased altruism evolves. Above the singularity, the adaptive process causes the rise of altruism up to the physiological bound. For species with high $\kappa$ ("strong linear" costs), the selfish state is evolutionarily attractive at any value of the mobility rate (Fig. 5D).

This pattern can be understood by comparing selective pressures (Figs. 4B, C). In the case of species with linear costs, the marginal benefit of altruism, $(1-\phi) \cdot \tilde{q}_{y \mid y}$, increases monotonously towards $\phi \cdot(1-\phi)$ as the altruistic investment becomes larger. If $\kappa$ is larger than this value, the marginal costs of altruism always oppose the evolution of altruism (Fig. 4C). Otherwise, in species with low mobility marginal benefits are sufficiently high in the selfish state to select for altruism (Fig. 4B); in species with high mobility, marginal benefits exceed marginal costs only at high investment in altruism, and selfishness is locally attractive. The mobility threshold, where the stability of selfishness switches from global repulsion to local attraction, is given by the mobility rate $m_{l}$ of a selfish population at which marginal benefits $(1-\phi) \cdot \tilde{q}_{y \mid y}$ and marginal costs $\kappa$ equalize: 


$$
m_{l}=b \frac{\phi \cdot(1-\phi)-\kappa}{v(\phi \cdot(1-\phi)-\kappa)+\kappa \cdot(1-\phi)} .
$$

This relation shows that among slowly reproducing organisms (small $b$ ), altruism may evolve only in species that exhibit little mobility. In species with very weak linear costs, the mobility threshold may not be observed, for it may exceed the critical value $(b-d) / v$ above which the population becomes non-viable (Appendix $3, \kappa<0.05$ in Fig. $5 \mathrm{~A})$. As a consequence, selfishness is invaded by altruism at any mobility rate smaller than this critical value, whereas at higher mobility, the evolution of decreasing altruism always drives the population to extinction (not shown).

\section{Accelerating Costs}

With accelerating costs, the cost of altruism is negligible compared to the benefits as long as the investment in altruism is not too high, and the selfish state is always invadable. This is in sharp contrast with predictions from well-mixed populations, in which selfishness is uninvadable even by only slightly altruistic mutants as soon as altruistic individuals incur a non-zero cost (Equation (A14) in Appendix 4). As altruistic phenotypes gain a foothold in the population, there are two possible outcomes depending on the combination of cost parameters.

Under a pattern of "slowly accelerating cost" (Fig. 5E), altruism rises toward a high evolutionary singularity in species with low mobility. With higher mobility the adaptive dynamics regime is bistable: the adaptive process converges to a high or a low singularity depending on the ancestral state. Extensive numerical explorations show that the altruism is always obligate (facultative) at the high (low) singularity. Under a pattern of "rapidly accelerating cost" (Fig. 5F), the adaptive dynamics converge monotonously to a low altruistic investment, whatever the ancestral state (including selfishness). The selected altruistic trait is found to correlate negatively with mobility. The evolved altruistic interactions shift from facultative to obligate as the cost parameters $\gamma$ and or $\kappa$ increase.

The analysis of selective pressures helps us to understand these results (Figs. 4D, E). With slowly accelerating costs, when mobility is low, the marginal benefits start high and increase slowly (Fig. 4D). Then mutant relatedness easily opposes the initially low but faster-growing marginal cost. The selective force that favors altruism keeps dominating as the investment in altruism increases, until the marginal costs and benefits of altruism balance each other, which occurs at a high value of altruism. At higher mobility rates, the initial level of relatedness is lower, yet it remains sufficient for altruism to invade (Fig. 4D). The increase of relatedness with altruism is slower, which causes the selective pressures to balance at a low-altruism singularity. Beyond this point, the negative pressure exerted by the cost grows smoothly, while the positive pressure catches up rapidly across a range of intermediate investments (Fig. 4D). This generates a second unstable singularity, above which the net selective pressure turns positive again and favors the increase of altruism until a third, attractive singularity is reached at high altruistic investment. With rapidly accelerating costs, the selective 
pressures balance at a low-altruism singularity (Fig. 4E). Above the singularity, the net selective pressure against altruism increases, whereas the positive ecological feedback through relatedness remains weak. Although in general there is no analytical expression for the singularities, the selected altruistic trait reaches a maximum value in the limit where mobility becomes very low, which is given explicitly by

$$
u_{\max }^{*}=\left[\frac{\phi(1-\phi)}{\kappa \gamma}\right]^{\frac{1}{\gamma-1}} \text {. }
$$

This maximum is independent of the organism's birth and death rates, and it decreases as the neighborhood size $n$ increases. In species that experience rapidly accelerating costs, the singularity smoothly rises toward $u_{\max }^{*}$ as mobility decreases. In contrast, species with slowly accelerating costs fall into two main categories: quasiselfish species, and obligatory altruistic species. In these obligatory altruistic species, the level of altruism is approximately equal to $u_{\max }^{*}$, and thus primarily depends on cost parameters and habitat connectedness.

\section{Evolutionary stability and large mutations}

The previous analysis based on the canonical equation assumes small mutational steps and does not yet address the potential invasibility of attractive states. A locally attractive singularity would give rise to evolutionary branching if it is invadable (Metz et al. 1996). Evolutionary stability is probed by inspecting pairwise invasibility plots (PIPs; Geritz et al. 1998) that display the sign of $s_{x}(y)$ as $u_{x}$ and $u_{y}$ vary throughout the trait space (Fig. 6). Mutation effects are actually small but not infinitesimal, and even large mutations may occur, albeit rarely. The PIPs also describe the invasion potential of mutants that may differ substantially from their resident progenitors.

For species with decelerating costs, the PIPs show that selfishness is locally uninvadable: a slightly altruistic phenotype may not thrive in a primeval egoistic world. However, large mutations can move the population out of the basin of attraction of the selfish state, thus allowing for the adaptive increase of altruism (Fig. 6A). Even starting from a purely selfish population, rare mutations of large effect, together with random drift in a finite size population, makes this occur with certainty, although the waiting time can be long. The case of species with linear costs is radically different: even very large mutations may not move the population out of the basin of attraction of the selfish state, which is thus globally evolutionary stable (Figs. 6B, C).

In species with accelerating costs, the PIPs indicate that the attractive singularities are uninvadable (Figs. 6D, E). Starting from any viable trait value, altruism gradually evolves toward the singularity, which is robust against invasion by any alternative mutant. Large mutations may fail to invade even if they occur in the direction of adaptation predicted by the selection derivative (Figs. 6D, E). This will cause the adaptive process to slow down, all the more as it approaches the singularity. The nontrivial zero contour of fitness flattens in response to increased mobility, indicating that this "evolutionary slowing down" (Dieckmann and Law 1996) should be more pronounced in more mobile species. 

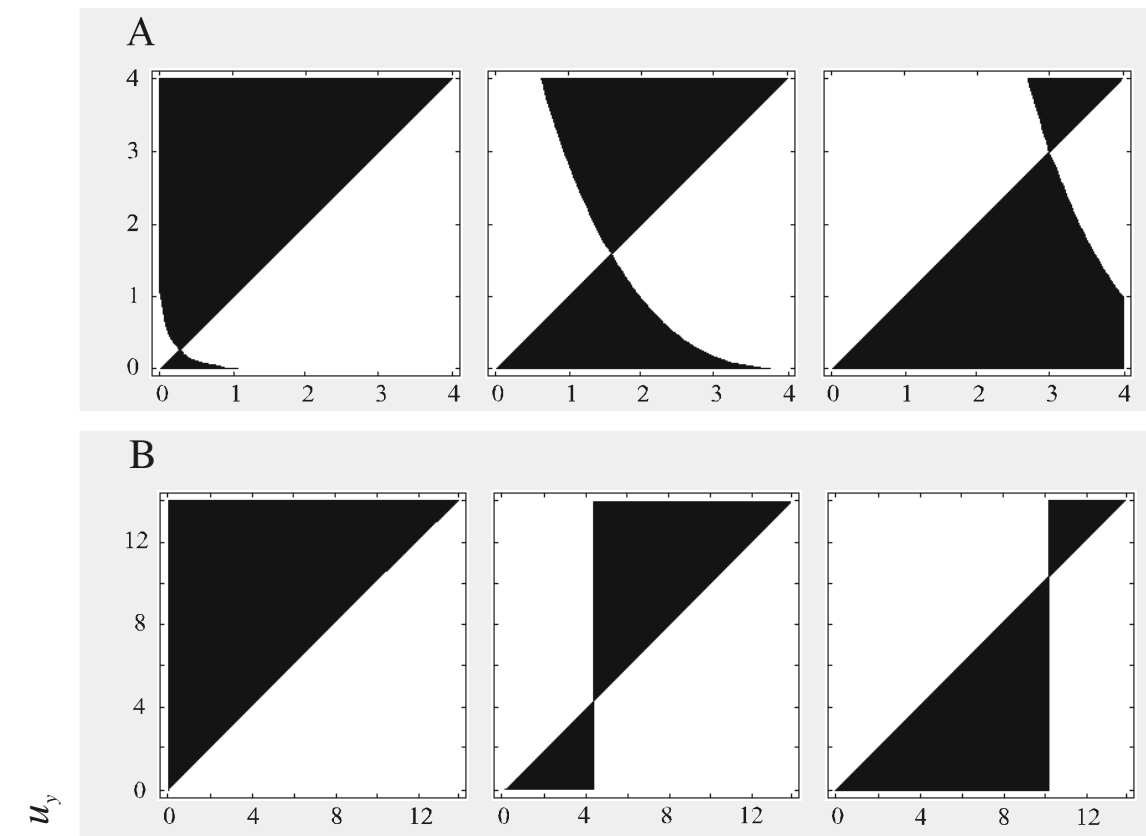

हี


Resident investment in altruism, $u_{x}$ 
F ig u r e 6 . Invasibility of altruism phenotypes, tested at different mobility rates. Each pair wise invasibility plot represents the sign and zero contour of spatial invasion fitness as a function of the resident (horizontal axis) and mutant (vertical axis) trait values. Black (white) areas indicate combinations of resident altruism and mutant phenotypes for which spatial invasion fitness $s_{x}(y)$ is positive (negative). Singularities lie at the intersection of the diagonal line $s_{x}(x)=0$ and non-trivial zero contour $s_{x}(y)=0$ with $y \neq x$. A singularity $x^{*}$ is locally uninvadable if for any $y$ in the vicinity of $x^{*} s_{x^{*}}(y)$ is negative. A singularity $x^{*}$ is attracting if the spatial invasion fitness is positive above the diagonal on the left of $x^{*}$ and below the diagonal on the right of $x^{*}$. A, Decelerating costs, with $m=0, m=4$ and $m=8$ from left to right. The singularity is attracting. A weak investment in altruism leads to selfishness by infinitesimal mutational steps, but the selfish state is invaded by large mutations. B, Weak linear costs with $m=0, m=4$ and $m=8$ from left to right. At low mobility, the increase of altruism is always favored. At high mobility, there is one repelling singularity. An initially small investment in altruism leads to selfishness by mutational steps of any size. C, Strong linear costs with $m=0$, $m=3$ and $m=6$ from left to right. Selfishness is attracting, and also locally and globally evolutionarily stable. D, Slowly accelerating costs with $m=0, m=7.5$ and $m=15$ from left to right. At low mobility, altruism converges to a high singularity that is uninvadable. For higher mobility, two evolutionary attracting singularities are separated by a repelling singularity; these attracting singularities are uninvadable by mutations of any size. E, Rapidly accelerating costs with $m=0, m=4.5$ and $m=9$ from left to right. The singularity is evolutionarily attracting and uninvadable by mutations of any size. The inspection of many generic pairwise invasibility plots led us to conclude that in our model the continuous evolution of altruism never undergoes evolutionary branching. All unspecified parameters in panels A to E are as in Fig. 2B to F, respectively.

\section{Polymorphic simulations}

Although our analytical investigation of the evolution of altruism incorporates salient features of the ecological and evolutionary processes, it also involves several important simplifications. We assume an infinite lattice size, and describe the ecological dynamics with the standard pair approximation (Appendix 2). The derivation of the fitness measure relies on the small frequency of mutants as they originate and on the assumption that that the build-up of the mutant's pseudo-equilibrium correlation structure is so fast that it can be regarded as instantaneous (Fig. 3). Furthermore, the deterministic description of the adaptive dynamics is an approximation for the mean path of a stochastic mutation selection-process (Dieckmann and Law 1996). Individualbased simulations that track the fate of each individual in the population (Appendix 1) provides a natural way to circumvent these limitations and can be used to test the robustness of our main findings.

Patterns of invasion can be probed by running a large number of stochastic simulations in which a single individual mutant arises in a stable, resident population (Fig. 7A). We observe a sharp increase of the mutant local frequency $q_{y \mid y}$ at low values of $p_{y}$ which corresponds to the rapid phase of convergence towards the pseudoequilibrium of the mutant correlation structure (i.e., $\tilde{q}_{y \mid y}$ ) predicted by the pair approximation (Fig. 3). 

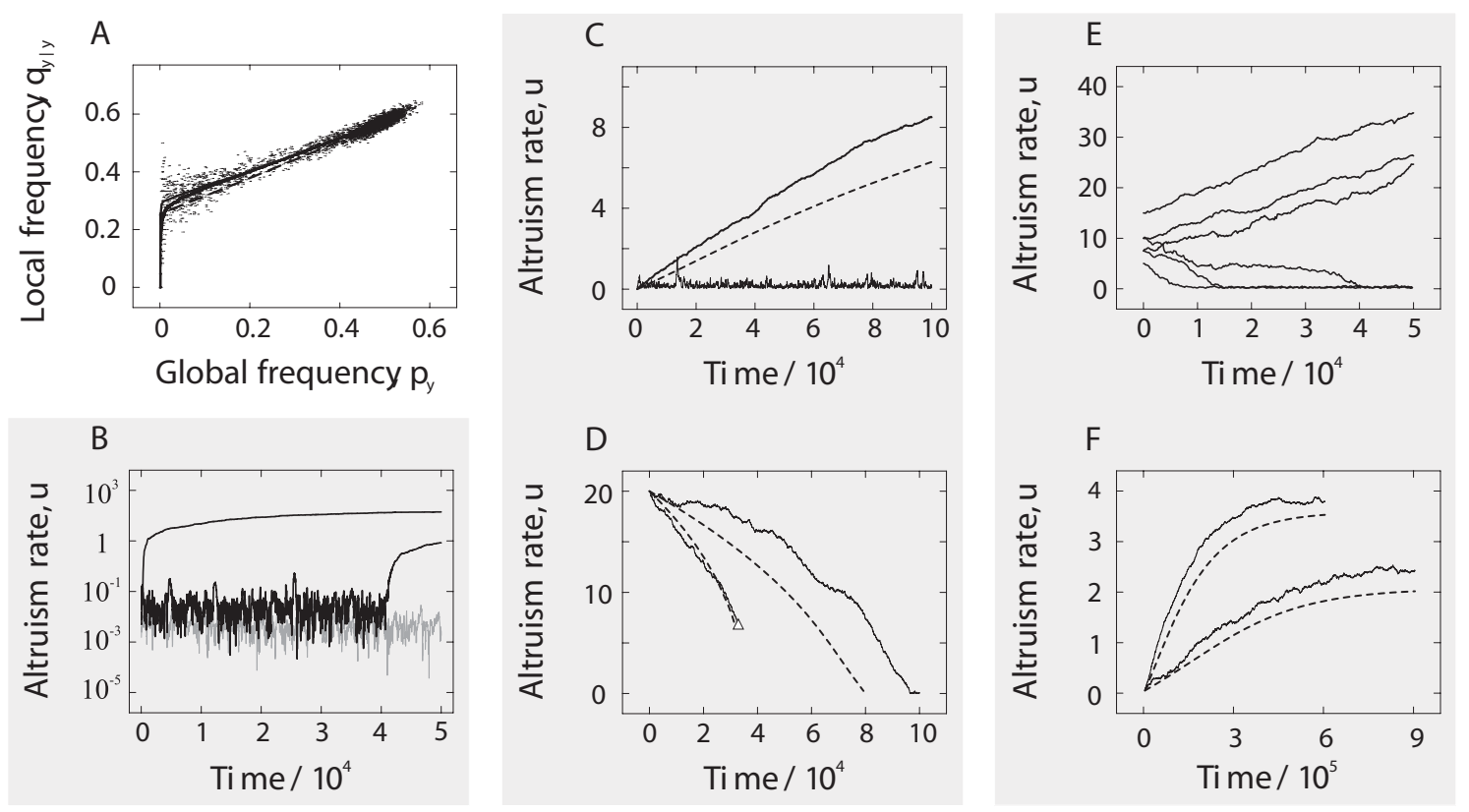

F ig u r e 1. Individual-based simulations of the mutation-selection process. A, Invasion dynamics. Deterministic dynamics (dashed curve) compared with stochastic simulations (continuous curve, mean of 1500 runs). Each dot gives the altruist local frequency $q_{y \mid y}$ and the altruist frequency $p_{y}$ value at one point in time for 6 independent simulations. Parameter values as in Fig. 2. B-F, Adaptive dynamics. B, Species with decelerating costs. Selfishness is locally uninvadable but can be invaded by largish mutants. When the mobility rate is low and mutational variance is large $(m=0, \sigma=0.5)$, selfishness is rapidly displaced by altruism (first rising black trajectory). The waiting time before altruism takes off is longer with reduced mutational variance ( $\sigma=0.1$, second rising black trajectory). At even lower mutational variance ( $\sigma=0.01$ ), the population remains selfish over the simulation time (gray trajectory). Keeping mutational variance constant but increasing mobility or decreasing mutation rate causes a similar increase of the waiting time for altruism to take off. Mutation rate: $k=0.01$. C, Species with weak linear costs. Average of 10 independent stochastic trajectories (continuous upper curve) against the deterministic prediction computed from Equation (7) (dashed curve): altruism invades selfishness at low mobility ( $m=0, k=0.1, \sigma=0.1$ ). Lower trajectory: evolutionary stability of selfishness under weak linear costs and high mobility despite large mutational variance $(m=6, k=0.05, \sigma=0.1)$. D, Species with strong linear costs. Average of 10 independent stochastic trajectories (continuous trajectories) compared to the deterministic prediction computed from Equation (7) (dashed curves). At low mobility, an initially altruistic population converges to selfishness (right-hand side of panel; $m=0, k=0.1, \sigma=0.02$ ). At high mobility, altruism decreases adaptively until the population hits the extinction boundary (left-hand side of panel; $m=10, k=0.1, \sigma=0.02$ ). E, Species with slowly accelerating costs and intermediate mobility. Starting from an ancestral altruistic investment $u_{x}=5$, trajectories converge to a quasi-selfish state whereas an ancestral state at $u_{x}=15$ leads to the adaptive increase of altruism toward a singularity. Starting from the same ancestral state, $u_{x}=8$ or $u_{x}=10$, stochastic trajectories either rise to a high singularity or decline toward quasi-selfishness (10 stochastic runs per starting condition). Parameter values: $m=15, k=0.05, \sigma=0.1$. F, Rapidly accelerating cost. Average of 10 independent stochastic trajectories (continuous curves) compared to the deterministic prediction (dashed curves), at low mobility ( $m=0$, upper curves, simulations run over 600,000 time units) and high mobility ( $m=6$, lower curves, simulation run over 900,000 time units). Mutation parameters: $k=0.01, \sigma=0.01$. The agreement between stochastic simulations and the deterministic approximation is satisfactory. In general, the deterministic approximation converges slower than the stochastic process and underestimates the adaptive altruistic investment. This may be due to small systematic errors introduced by the standard pair approximation. Unspecified parameters as in Fig. 2B to F, respectively. 
The evolutionary patterns predicted by the canonical equation can be tested by running individual-based simulations of the mutation-selection process in which the assumption that mutants arise one at a time is relaxed, and averaging over a set of simulations (Figs. 7B to F). In species with decelerating costs, we predict that selfishness is invaded by altruistic phenotypes after a potentially long waiting time. Individual-based simulations confirm this prediction and show that the adaptive increase of altruism starts earlier when mutational effects are larger (Fig. 7B). Possibly, for very small mutational effects, the corresponding waiting time may be too long for being observed in simulations of feasible duration. In species with weak linear costs, individual-based simulations confirm both the adaptive increase of altruism at low mobility and the evolutionary stability of selfishness at high mobility, even when very large mutations are feasible (Fig. 7C). In species with strong linear costs, convergence to selfishness or evolutionary suicide, depending on mobility, occurs as predicted by the canonical equation (Fig. 7D). Species with slowly accelerating costs are characterized by two clear-cut patterns, namely quasi-selfishness versus high altruism. At intermediate mobility, the adaptive dynamics bistability is confirmed by individualbased simulations run from different ancestral conditions (Fig. 7E). This implies that alternative adaptive investments can be reached for identical life-history profiles, due to different ancestral states or to contingent events during evolutionary history. Notice also that quasi-selfish states are practically indistinguishable from the stable selfish state of a stochastic mutation-selection process. In species characterized by rapidly accelerating costs the individual-based simulations closely match the predictions (Fig. 7F). The negative correlation between selected altruism and mobility expected in this case is confirmed.

Random lattices (featuring randomly assigned connections between sites) have been proposed for the purpose of modeling social networks (Rand 1999). The alternative of a regular habitat geometry, where interactions are limited to the geographically closest sites, compromises the use of the standard pair approximation to derive correlation equations for the population dynamics (van Baalen 2000). Individual-based simulations involving a regular square lattice indicate that our main findings are not altered qualitatively whereas selected trait values tend to be higher (results not shown).

\section{Discussion}

We study the adaptive dynamics of a quantitative trait that measures the individual investment in altruism. The habitat is constant and homogeneous, but selective pressures arise from the phenotypic heterogeneity of the population. Altruism evolves from selfishness under a gradual kin selection process. This pattern is similar to the continuous evolution of cooperative investment observed in a spatial evolutionary game (Killingback et al. 1999). However, our model is not restricted to accelerating costs and pure viscosity, which would make up the most favorable case for the rise of altruism in a selfish population. In fact, we show that the qualitative and quantitative features of the adaptive evolution of altruism depend on the patterns of the cost and their interactions with mutation, individual mobility and habitat structure. Even when an accelerating cost pattern allows altruism to evolve easily from selfishness (Killingback et al. 1999), the 
adaptive increase of altruism appears to be often halted at very low levels. In contrast, when the cost pattern is decelerating, the selfish state will usually be displaced upon the occurrence of rare mutations of large effects.

\section{Kin Selection, Cooperation and Competition}

The conceptual path followed here to define and measure fitness differs from the usual approach of kin selection theory (Frank 1998; Michod 1999 for reviews), although the results from both angles can be formulated in similar terms. Starting from demographic and behavioral processes operating at the level of individuals and their neighbors, we follow on from van Baalen and Rand (1998) to define fitness as the invasion exponent of a system of correlation equations for the spatial dynamics of a mutant population. This notion extends the concept of invasion fitness defined for well-mixed populations (Metz et al. 1992; Rand et al. 1994; Ferrière and Gatto 1995). In our model, invasion fitness is found to compound the per capita intrinsic growth rate and the per capita "neighbor-modulated" growth rate (Frank 1998) that accounts for the respective effects of competitive and cooperative interactions between a mutant focal individual and mutant versus resident neighbors.

In the terminology of kin selection, this invasion fitness is analogous to a "direct fitness function" from which one can derive a direct fitness gradient and decipher the selective pressures that operate on the trait under consideration (Frank 1998). We identify three selective pressures acting on altruism: the direct physiological cost to the individual, the indirect beneficial effect of altruistic interactions, and the indirect negative effect of competition for space. According to the analysis of Taylor (1992a, 1992b) and Queller (1994), spatial kin selection models raise a critical difficulty for the evolution of cooperation, because costs of competing for space with relatives exactly cancel out the benefits of altruism. Similarly, Wilson et al. (1992) concluded "local population regulation often, if not always, cancels the effects of relatedness". We come here to the opposing conclusion that the cost of competing for space with relatives exerts a negligible selective pressure against altruism, which reemphasizes that kin selection is effective at explaining the evolution of unconditional altruism.

This result holds independently of the level of individual mobility; and therefore cannot be attributed to relaxing the "pure viscosity" hypothesis of traditional kin selection models, according to which only offspring are dispersed (Hamilton 1964). Also, the explanation involving some disjunction of the social and economic neighborhoods, whereby social partners could be more related than competitors (Queller 1992, 1994), does not apply here. Indeed, the expression for the invasion fitness shows clearly that both negative density dependence (competition) and positive density dependence (social interactions) are only determined by the fraction of occupied sites in the same neighborhood. Thus social and economic neighborhoods coincide exactly.

The key feature of our model therefore lies in the assumption that the habitat offers empty sites for the local spread of altruistic mutants. This occurs because the demographic death process keeps reopening space in the neighborhood of any focal individual. Consequently, the population does not reach a steady state of saturation, and the selective pressure generated by local competition remains negligible. Such a 
dominant effect of the availability of free space on the evolution of altruism was anticipated by Taylor (1992b) and Queller $(1992,1994)$ who introduced the notion of "population elasticity" to refer to it. In a recent study of a contest of selfishness versus altruism, Mitteldorf and Wilson (2000) included a similar effect as generated by environmental stochasticity, and came to the same conclusion. However, these authors assumed an initial mixture of egoists and altruists instead of a single mutant in a resident background population, which does not address the crucial phase of the invasion process.

Whether it is demographic stochasticity or environmental stochasticity that underlies the site-opening process is unlikely to make much difference as long as there is no feedback of the adaptive trait dynamics on that stochastic process. This is the case both in our model and in the study by Mitteldorf and Wilson (2000) because the process of site opening amounts to a form of individual mortality whereas the altruistic trait affects fecundity. In a different setting where altruism would impact on the individual mortality rate, such a feedback could exist depending on whether sites are opened by demographic or environmental stochasticity. The adaptive increase of altruism would reduce the death rate, hence the rate of site opening by demographic stochasticity. As a consequence, the selective pressure of local competition against altruism would be enhanced, as can be demonstrated by an extension of our analysis (results not shown) and by the studies of Nakamaru et al. (1997, 1998) and Taylor and Irwin (2000). By contrast, the rate of site opening due to environmental stochasticity could remain independent of the adaptive change in altruism. Environmental stochasticity might then become critical for the evolution of altruism.

Because the selective pressure resulting from competition for open space turns out to be negligible, the criterion for mutant invasion amounts simply to comparing the marginal physiological cost of altruism to the marginal benefit withdrawn from interaction with the mutant's own kind. This is a variant of Hamilton's rule (Hamilton 1964), where the coefficient of relatedness is given by the local frequency of mutants neighboring a focal mutant during the invasion process. That the probability for the recipient of a focal mutant's act also to be a mutant is the appropriate definition of relatedness has already been shown in kin selection models involving pairwise interactions (Day and Taylor 1998, Frank 1998). However, such kin selection models assume that relatedness is constant and that the phenotype of a mutant has no effect on relatedness, which obviously cannot be true when invasion is a dynamical process and altruism impacts the distribution of individuals across space. The approach first advocated by van Baalen and Rand (1998) and followed up in this study shows that the same concept of relatedness holds nonetheless. In effect, relatedness is a dynamical variable for which the relevant equilibrium value can be expressed as a function of basic features of the organism's life cycle and behavior (also see Ferrière and Michod 1995, 1996; Hutson and Vickers 1995; van Baalen and Rand 1998). 


\section{Adaptive Patterns of Altruism}

The pattern of physiological costs, individual mobility, the mutation process and habitat connectedness all interact to determine the adaptive dynamics of altruism (Table 2). The consideration of decelerating costs is more relevant to study the rise of altruism from selfishness. In species with decelerating costs, altruism is under the most stringent conditions to evolve from selfishness. However, once altruism is established by the mutation-selection process, the population invariably evolves toward the physiologically maximum investment. In contrast, the assumption of accelerating costs is more relevant to study the determinants of adaptive variation in altruism. In species with accelerating costs, altruism evolves right away from selfishness (Killingback et al. 1999); but contrary to the case of decelerating costs, the evolutionary endpoint is predicted to vary according to the physiological and ecological parameters.

Following on from this dichotomy, an important result is that generically any ancestral selfish population will evolve some degree of altruism. In the case of accelerating costs, the evolution of altruism is not influenced by the mutation process. On the contrary, assuming that in the primeval selfish state the cost of altruism is decelerating, the adaptive initiation of altruism depends primarily on the mutation process. We found that higher mutation frequency and larger mutational steps decrease the waiting time for altruism to evolve. For a given mutation process, the waiting time also increases with mobility, as higher mobility carries a larger direct cost and lowers the indirect benefit of altruism. The study of a linear cost function, which can be interpreted as a degenerate case in between decelerating or accelerating cost patterns, pinpoints the fallacious consequences that result from restricting attention to such a simple case (e.g., Roberts and Sherrat 1998). Incorporating a strong linear cost in the model completely hides the potential effect of the genetic process on the displacement of selfishness. The assumption of weak linear costs yields a type of bistable adaptive dynamics similar to the case of decelerating costs. However, large mutations have no effect in this case.

Mobility is expected to be an important factor of adaptive variation in altruism, as shown by the study of accelerating costs of altruism. First, mobility is found to impact on the speed of the adaptive process - the altruistic trait evolves at a slower pace among more mobile individuals. Second, mobility is a significant determinant of the evolutionary endpoint. In species with rapidly accelerating costs, the selected altruistic trait value correlates negatively with mobility. In species with slowly accelerating costs, mobility has a profound qualitative effect. High altruistic investments evolve in species with low mobility whereas quasi-selfishness evolves in species with high mobility. The finding of altruism being associated with low mobility is in line with previous insights into the evolution of cooperation in the Iterated Prisoner's Dilemma game (Dugatkin and Wilson 1991; Enquist and Leimar 1993; Hutson and Vickers 1995; Ferrière and Michod 1995, 1996), although the mechanisms involved are different. In these studies, mobility opposes the evolution of altruism by reducing the probability of repeated interactions between the same partners. On the empirical side, many independent studies have related the evolution of complex social systems with reduced mobility. For 
I a b le 2 , Overview of results derived in this paper.

\begin{tabular}{|c|c|c|}
\hline Physiological cost & Adaptive dynamics & $\begin{array}{l}\text { Evolutionary outcome from a } \\
\text { selfish ancestral state }\end{array}$ \\
\hline Decelerating costs & Bistability & $\begin{array}{l}\text { - Selfishness always displaced if } \\
\text { finite mutations occur } \\
\text { - Waiting time for the adaptive rise } \\
\text { of altruism increases as mutation } \\
\text { rate or mutational variance } \\
\text { decreases, or mobility increases } \\
\text { - Evolution toward physiologically } \\
\text { maximum investment in altruism }\end{array}$ \\
\hline \multirow[t]{2}{*}{ Weak linear costs } & $\begin{array}{l}\text { Monotonic increase at } \\
\text { low mobility }\end{array}$ & $\begin{array}{l}\text { - Evolution toward physiologically } \\
\text { maximum investment in altruism } \\
\text { if mobility is low }\end{array}$ \\
\hline & $\begin{array}{l}\text { Bistability at high } \\
\text { mobility }\end{array}$ & $\begin{array}{l}\text { - Persistent selfishness if mobility } \\
\text { is high }\end{array}$ \\
\hline Strong linear costs & Monotonic decrease & - Persistent selfishness \\
\hline \multirow{2}{*}{$\begin{array}{l}\text { Slowly accelerating } \\
\text { costs }\end{array}$} & $\begin{array}{l}\text { Monotonic } \\
\text { convergence at low } \\
\text { mobility }\end{array}$ & $\begin{array}{l}\text { Evolution toward high altruistic } \\
\text { investment if mobility is low }\end{array}$ \\
\hline & $\begin{array}{l}\text { Bistability at high } \\
\text { mobility }\end{array}$ & $\begin{array}{l}\text { Evolution toward quasi- } \\
\text { selfishness if mobility is high }\end{array}$ \\
\hline $\begin{array}{l}\text { Rapidly accelerating } \\
\text { costs }\end{array}$ & $\begin{array}{l}\text { Monotonic } \\
\text { convergence }\end{array}$ & $\begin{array}{l}\text { Evolution toward low altruistic } \\
\text { investment, correlating } \\
\text { negatively with mobility }\end{array}$ \\
\hline
\end{tabular}

example, the emergence of cooperative breeding in birds may have been driven by delayed dispersal in a context of intense competition for space ("habitat saturation" hypothesis; Emlen 1982, 1997). Also, African mole-rat populations exhibit strong levels of philopatry, and the eusocial species of this mammal group occur in the harshest habitats, namely arid zones where benefits of group-living are high and dispersal is low (Jarvis et al. 1994; Spinks et al. 2000).

In species with slowly accelerating costs and low mobility, most variations of altruism between species are expected in response to differences in physiological costs and the degree of habitat connectedness. Our model outlines the importance of 
describing and measuring these parameters (Heinsohn and Legge 1999). Controlled experiments including the analysis of a broad range of levels of investment in altruism would enhance our knowledge of the shape and values of costs of altruism. In reality, the costs of cooperation may impact on different life history traits at different periods of the individual lifetime, and these experiments would require multivariate approaches and long-term studies.

In contrast, in species with slowly accelerating costs and high mobility, quasiselfishness is expected to evolve. This state is eventually indistinguishable from a stable selfish population in which slightly altruistic phenotypes would chronically spread by mutation and drift. High altruistic investments could still be observed by the phylogenetic conservation of an ancestrally altruistic state while a slow environmental change favors an increase in mobility (Fig. 5E). High altruistic investment would have evolved under the early low mobility, and be preserved throughout the subsequent environmental increase of mobility. In support of the role of phylogenetic conservation, recent comparative analyses of sociality have demonstrated a phylogenetic component of sociality in birds (Edwards and Naeem 1993) and different groups of arthropods (Crespi 1996; Wcislo and Danforth 1997). High levels of investment in sociality seem to be maintained in more various ecological contexts once they evolve from cooperative ancestors. Possibly, a slow, gradual environmental change could even cause the loss of altruistic behavior at very high mobility through the catastrophic extinction of the population (Fig. 5E). An environmentally driven loss of sociality may provide another element of explanation for the uneven distribution of social species across taxa (Velicer et al. 1998).

Our analysis highlights the critical importance of mobility, which we modeled as a fixed parameter. This simplifying assumption may apply to species in which mobility is strongly constrained by the environment, the developmental program, or the genetic system. Otherwise, mobility and altruism should be entangled in a co-adaptive process, the dynamics of which will be investigates in a forthcoming paper. 


\section{References}

Axelrod, R., and W. D. Hamilton. 1981. The evolution of cooperation. Science 211:1390-1396.

Bourke, A. F. G. 1997. Sociality and kin selection in insects. Pp. 203-227 in J. R. Krebs and N. B. Davies, eds. Behavioural ecology: an evolutionary approach. Blackwell, Oxford.

Cockburn, A. 1998. Evolution of helping behavior in cooperatively breeding birds. Ann. Rev. Ecol. Syst. 29:141-177.

Crespi, B. J. 1996. Comparative analysis of the origins and losses of eusociality: causal mosaics and historical uniqueness. Pp. 253-287 in E. Martins, ed. Phylogenies and the comparative method in animal behavior. Oxford Univ. Press, Oxford.

Day, T., and P. D. Taylor. 1998. Unifying genetics and game theoretic models of kin selection for continuous traits. J. Theor. Biol. 194:391-407.

Dieckmann, U., and R. Law. 1996. The dynamical theory of coevolution: a derivation from stochastic ecological processes. J. Math. Biol. 34:579-612.

Dieckmann, U., and R. Law. 2000. Relaxation projections and the methods of moments. Pp. 412-455, in U. Dieckmann, R. Law, and J. A. J. Metz, eds. The geometry of ecological interactions: simplifying spatial complexity. Cambridge Univ. Press, Cambridge.

Doebeli, M., and N. Knowlton. 1998. The evolution of interspecific mutualisms. Proc. Natl. Acad. Sci. 95:8676-8680.

Dugatkin, L. A., and D. S. Wilson. 1991. ROVER: a strategy for exploiting cooperators in a patchy environment. Am. Nat. 138:687-701.

Edwards, S. V., and S. Naeem. 1993. The phylogenetic component of cooperative breeding in perching birds. Am. Nat. 141:754-789.

Emlen, S. T. 1982. The evolution of helping. I. An ecological constraints model. Am. Nat. 119:29-39.

Emlen, S. T. 1997. Predicting family dynamics in social vertebrates. Pp. 228-252 in J. R. Krebs and N. B. Davies, eds. Behavioural ecology: an evolutionary approach. Blackwell, Oxford.

Enquist, M., and O. Leimar. 1993. The evolution of cooperation in mobile organisms. Anim. Behav. 45:747-757.

Ferrière, R., and M. Gatto. 1995. Lyapunov exponents and the mathematics of invasion in oscillatory or chaotic populations. Theor. Pop. Biol. 48:126-171.

Ferrière, R., and R. E. Michod. 1995. Invading wave of cooperation in a spatial iterated prisoner's dilemma. Proc. Roy. Soc. London B 259:77-83. 
Ferrière, R., and R. E. Michod. 1996. The evolution of cooperation in spatially heterogeneous populations. Am. Nat. 147:692-717.

Ferrière, R., and J.-F. Le Galliard. 2001. Invasion fitness and adaptive dynamics in spatial population models. Pp. 57-79 in J. Clobert, E. Danchin, A. A. Dhondt and J. D. Nichols, eds. Dispersal. Cambridge Univ. Press, Cambridge.

Frank, S. A. 1998. Foundations of social evolution. Princeton Univ. Press, Princeton.

Geritz, S. A. H., E. Kisdi, G. Meszéna, and J. A. J. Metz. 1998. Evolutionary singular strategies and the adaptive growth and branching of the evolutionary tree. Evol. Ecol. 12:35-57.

Gillespie, T. D. 1976. A general method for numerically simulating the stochastic time evolution of coupled chemical reactions. J. Comp. Phys. 22:403-434.

Hamilton, W. D. 1964. The genetical evolution of social behaviour I, II. J. Theor. Biol. $7: 1-52$.

Heinsohn, R., and A. Cockburn. 1994. Helping is costly to young birds in cooperatively breeding white-winged coughs. Proc. Roy. Soc. London B 256:299-303.

Heinsohn, R., and S. Legge. 1999. The costs of helping. TREE 14:53-57.

Hutson, V., and G. T. Vickers. 1995. The spatial struggle of tit-for-tat and defect. Phil. Trans. Roy. Soc. Lond. B 348:393-404.

Iwasa, Y. 2000. Lattice models and pair approximation in ecology. Pp. 227-251 in U. Dieckmann, R. Law, and J. A. J. Metz, eds. The geometry of ecological interactions: simplifying spatial complexity. Cambridge Univ. Press, Cambridge.

Jarvis, J. U. M., M. J. O'Riain, N. C. Bennett and P. W. Sherman. 1994. Mammalian eusociality: a family affair. TREE 9:47-51.

Killingback, T., M. Doebeli, and N. Knowlton. 1999. Variable investment, the Continuous Prisoner's Dilemma, and the evolution of cooperation. Proc. Roy. Soc. London B 266:1723-1728.

Koella, J. 2000. The spatial spread of altruism versus the evolutionary response of egoists. Proc. Roy. Soc. London B 267:1979-1985.

Marrow, P., R. Law, and C. Cannings. 1992. The coevolution of predator-prey interactions: ESSs and Red Queen dynamics. Proc. Roy. Soc. London B 250:133141.

Matsuda, H., N. Ogita, A. Sasaki, and K. Sato. 1992. Statistical mechanics of population: the lattice Lotka-Volterra model. Progress in Theoretical Physics 88:1035-1049.

Maynard-Smith, J., and E. Szathmary. 1995. The major transitions in evolution. Oxford Univ. Press, Oxford.

Metz J. A. J., R. M. Nisbet, and S. A. H. Geritz. 1992. How should we define fitness for general ecological scenarios? TREE 7:198-202. 
Metz J. A. J., S. A. H. Geritz, G. Meszéna, F. J. A. Jacobs, and J. S. van Heerwaarden. 1996. Adaptive dynamics, a geometrical study of the consequences of nearly faithful reproduction. Pp. 183-231 in S. J. van Strien and S.M. Verduyn Lunel, eds. Stochastic and spatial structures of dynamical systems, North Holland, Amsterdam.

Michod, R. E. 1999. Darwinian dynamics, evolutionary transitions in fitness and individuality. Princeton Univ. Press, Princeton.

Mitteldorf, J., and D. S. Wilson. 2000. Population viscosity and the evolution of altruism. J. Theor. Biol. 204:481-496.

Nakamaru, M., H. Matsuda, and Y. Iwasa. 1997. The evolution of cooperation in a lattice-structured population. J. Theor. Biol. 184:65-81.

Nakamaru, M., H. Nogami, and Y. Iwasa. 1998. Score-dependent fertility model for the evolution of cooperation in a lattice. J. Theor. Biol. 194:101-124.

Nowak, M. A., and R. May. 1992. Evolutionary games and spatial chaos. Nature 359:826-829.

O'Riain, M. J., J. U. M. Jarvis, and C. G. Faulkes. 1996. A dispersive morph in the naked mole-rat. Nature 380:619-521.

Pusey, A. E., and C. Packer. 1997. The ecology of relationships. Pp. 254-283 in J. R. Krebs and N. B. Davies, eds. Behavioural ecology: an evolutionary approach. Blackwell, Oxford.

Queller, D. C. 1992. Does population viscosity promote kin selection? TREE 10:322324.

Queller, D. C. 1994. Genetic relatedness in viscous populations. Evol. Ecol. 8:70-73.

Rand, D. A., H. B. Wilson, and J. M. McGlade. 1994. Dynamics and evolution: evolutionarily stable attractors, invasion exponents and phenotype dynamics. Phil. Trans. Roy. Soc. Lond. B 343:261-283.

Rand D. A. 1999. Correlation equations and pair-approximations for spatial ecologies. Pp. 100-142 in J. M. McGlade, ed. Advanced ecological theory: advances in principles and applications. Blackwell, Oxford.

Roberts, G., and T. N. Sherrat. 1998. Development of cooperative relationships through increasing investment. Nature 394:175-179.

Spinks, A. C., J. U. M. Jarvis, and N. C. Bennett. 2000. Comparative patterns of philopatry and dispersal in two common mole-rats populations: implications for the evolution of mole-rat sociality. J. Anim. Ecol. 69:224-234.

Strassmann, J. E., Y. Zhu, and D. Queller. 2000. Altruism and social cheating in the social amoeba Dictyostelium discoideum. Nature 408:965-967.

Taylor, P. D. 1992a. Inclusive fitness in a homogeneous environment. Proc. Roy. Soc. London B 249:299-302. 
Taylor, P. D. 1992b. Altruism in viscous populations - an inclusive fitness approach. Evol. Ecol. 6:352-356.

Taylor, P. D. and A. J. Irwin. 2000. Overlapping generations can promote altruistic behavior. Evolution 54:1135-1141.

Trivers, R. L. 1971. The evolution of reciprocal altruism. Quat. Rev. Biol. 46:35-57.

van Baalen, M., and R. A. Rand. 1998. The unit of selection in viscous populations and the evolution of altruism. J. Theor. Biol. 193:631-648.

van Baalen, M. 2000. Pair approximations for different spatial geometries. Pp. 359-387 in Dieckmann, U., R. Law, and J. A. J. Metz, eds. The geometry of ecological interactions: simplifying spatial complexity. Cambridge Univ. Press, Cambridge.

Velicer, G. J., L. Kroos, and R. E. Lenski. 1998. Loss of social behaviors by Myxococcus xanthus during evolution in an unstructured environment. Proc. Natl. Acad. Sci. USA 95:12376-12380.

Velicer, G. J., L. Kroos, and R. E. Lenski. 2000. Developmental cheating in the social bacterium Myxococcus xanthus. Nature 404:598-600.

Wcislo, W. T., and B. N. Danforth. 1997. Secondarily solitary: the evolutionary loss of social behavior. TREE 12:468-474.

Wilson, D. S., G. Pollock, and L. E. Dugatkin. 1992. Can altruism evolves in purely viscous populations? Evol. Ecol. 6:331-341. 


\section{Appendix 1 - Polymorphic individual-based model}

Here we describe the stochastic, individual-based model of polymorphic population dynamics that forms the basis of our study. The habitat structure is generated by randomizing the edges of a regular, square lattice of $30 \times 30$ sites with Moore neighborhoods (which, locally, gives a so-called Cayley tree or Bethe lattice). The state of any site is either empty or occupied by a type $i$ individual with a certain phenotype $u_{i}$. The demographic parameters at time $t$ of such an individual located at $z$ are the birth rate

$$
b_{i}(z, t)=\left[b(m)+\sum_{k=1}^{n} \frac{u_{k}}{n}-C\left(u_{i}\right)\right] \frac{n_{0 \mid i}(z, t)}{n},
$$

the intrinsic death rate $d$ and the mobility rate

$$
m_{i}(z, t)=m \frac{n_{0 \mid i}(z, t)}{n}
$$

where $k$ (varying from 1 to $n$ ) labels each neighboring site, $u_{k}$ is the altruistic investment of a neighbor or zero if that site is empty at that moment, and $n_{0 \mid i}(z, t)$ denotes the number of empty sites neighboring a type $i$ individual located at $z$ at time $t$. The local birth rate and movement rate are multiplied by the proportion of empty sites within the neighborhood.

The simulation starts by distributing individuals of an ancestral phenotype randomly over half of the lattice. Mutations generate variability with a probability $k$ per birth event. The mutant phenotype is obtained by adding a mutation effect drawn randomly from a normal probability distribution, with zero mean and mutational variance $\sigma^{2}$. When a negative investment in altruism is produced, the mutant phenotype is reset to selfishness. We use the so-called minimal process method (Gillespie 1976) to simulate the time-continuous stochastic dynamics of the population. This means that the waiting time between two events is drawn form an exponential distribution with the inverse of its mean given by the total sum of event rates per unit time. Typically the completion of such a stochastic simulation requires around a day of computation on the fastest personal computer available at the time this work was conducted.

\section{Appendix 2 - Spatial population dynamics}

Here, we derive an analytical model of the polymorphic population dynamics. The frequency of occupied sites varies through time along with the neighborhood configuration. The configuration of the neighborhood of a focal individual is given by the states of all pairs containing that individual, which themselves typically depend on the states of higher-order structures that contain those pairs (triplets, quadruplets, and so on). This kind of dependence cascades through all orders and spatial scales. Therefore, a 
full description of the lattice dynamics requires an infinite hierarchy of dynamical equations, also called correlation equations (Dieckmann and Law 2000). The system, however, can be closed at any order of description by making use of appropriate approximations. We close the system at the order of pairs with the standard pair approximation (Matsuda et al. 1992, Ferrière and Le Galliard 2001). This is the simplest approximation as it assumes that third-order correlations just vanish, and it has been used to construct correlation equations for spatial games (Nakamaru et al. 1997) and social interactions (Matsuda et al. 1992).

We first describe the temporal dynamics of the frequency of occupied sites. We assume that a fixed number of phenotypes are present in the population initially. We use the indices $i$ and $j$ to label phenotypes, and more generally the indices $k$ or $l$ to designate the state of a site, including the empty state 0 . We proceed by averaging the local birth rate (A1) over the lattice, which after some algebra yields the average birth rate of a type $i$ individual at time $t$ :

$$
\bar{b}_{i}(t)=\left[b(m)+\sum_{j} u_{j}(1-\phi) q_{j \mid i}(t)-C\left(u_{i}\right)\right] q_{0 \mid i}(t),
$$

where $q_{j \mid i}(t)$ is the local frequency of phenotype $j$ around a focal $i$ individual at time $t$, the sum is taken over all phenotypes in the population and $\phi=1 / n$. The mean change in the frequency of the $i$ phenotype at time $t$ during an infinitesimal time step $d t$ then is

$$
\frac{d p_{i}(t)}{d t}=\left(\bar{b}_{i}(t)-d\right) p_{i}(t)=\lambda_{i}(t) p_{i}(t) .
$$

We now describe the temporal dynamics of the local frequency of sites. As local frequencies are simply determined by pair frequencies according to

$$
q_{k \mid i}(t)=p_{k i}(t) / p_{i}(t)
$$

where $p_{k i}(t)$ designates the frequency of $k i$ pairs at time $t$, it is enough to describe the dynamics of the frequencies of pairs involved in (A5) to obtain equations for the local frequencies involved in (A4). An inventory of all different events that may affect any type of pairs at time $t$ yields three elementary fluxes (van Baalen and Rand 1998, Rand 1999). The first is the average per capita input rate of a type $i$ into a $0 j$ pair with $j \neq i$ :

$$
\alpha_{i j}(t)=(1-\phi)\left(\bar{b}_{i 0}(t)+m\right) q_{i \mid 0 j}(t)=\alpha_{i j}^{\prime}(t) q_{i \mid 0 j}(t) .
$$

The second flux corresponds to the average per capita input rate of a type $i$ into an $0 i$ pair:

$$
\beta_{i}(t)=\phi \bar{b}_{i 0}(t)+(1-\phi)\left(\bar{b}_{i 0}(t)+m\right) q_{i \mid 0 i}(t) .
$$

The third flux is the average per capita output rate of a type $i$ from an $i j$ pair:

$$
\delta_{i j}(t)=d+(1-\phi) m q_{0 \mid i j}(t) .
$$


The term $\bar{b}_{i 0}(t)$ involved in these fluxes is the average birth rate of a type $i$ individual inside a $i 0$ pair, given by

$$
\bar{b}_{i 0}(t)=\left(b(m)+\sum_{j} u_{j}(1-\phi) q_{j \mid i 0}(t)-C\left(u_{i}\right)\right) .
$$

From equations (A7), (A8) and (A9), we can obtain the dynamics of the frequencies for the three general types of pairs, $0 j, i j$ and $j j$. Unfortunately, the dynamics of these pair frequencies depend on higher-order configurations because $q_{k \mid i l}=p_{k i l} / p_{i l}$. Using the standard pair approximation, we assume that $q_{k \mid i l}=q_{k \mid i}$, which gives

$$
\begin{aligned}
& \frac{d p_{0 j}(t)}{d t}=\left(\alpha^{\prime}{ }_{j}(t) q_{0 \mid 0}(t)-\left(\beta_{j}(t)+\delta_{j}(t)\right)\right) p_{0 j}(t)+\sum_{k \neq(0, j)} \delta_{k}(t) p_{k j}(t)+\delta_{j}(t) p_{j j}(t) \\
& \frac{d p_{i j}(t)}{d t}=\left(\alpha_{i}(t)+\alpha^{\prime}{ }_{j}(t) q_{0 \mid 0}(t)\right) p_{0 j}(t)-\left(\delta_{j}(t)+\delta_{i}(t)\right) p_{i j}(t), \quad i \neq(0, j) \\
& \frac{d p_{j j}(t)}{d t}=2 \beta_{j}(t) p_{0 j}(t)-2 \delta_{j}(t) p_{j j}(t)
\end{aligned}
$$

\section{Appendix 3 - Monomorphic population}

A monomorphic version of the analytical model can be derived from (A4) and (A10). We assume there is only one phenotype and we denote by $x$ the state of an occupied site. We are interested here in the feasible population equilibria. According to equation (A4), the non-trivial population equilibrium $\bar{q}_{0 \mid x}$ must satisfy the quadratic equation

$$
\left(\left(b(m)+u_{x}(1-\phi)\left(1-\bar{q}_{0 \mid x}\right)-C\left(u_{x}\right)\right) \bar{q}_{0 \mid x}-d=0 .\right.
$$

The spatial population is also characterized by a second, independent statistic, $\bar{q}_{0 \mid 0}$. From (A10) $\bar{q}_{0 \mid 0}$ satisfies

$$
\bar{q}_{0 \mid 0}=\delta_{x} / \alpha_{x}^{\prime} .
$$

We now analyze the viability and stability of the equilibria if $b$ is sufficiently larger than $d$. The resident population is non-viable when no real solution exists for $\bar{q}_{0 \mid x}$, which gives a first extinction boundary $\Delta=0$ and the extinction domain $\Delta<0$ (where $\Delta$ denotes the discriminant of the quadratic Equation (A11)). In the area where $\Delta>0$, there are two sub-domains. In the sub-domain defined by $b(m)-d-C\left(u_{x}\right)>0$, the maximal root of (A11) corresponds to a viable equilibrium that is globally stable. In the disjunct sub-domain of obligate altruism, this solution is locally stable and coexists with the locally stable, trivial solution. A saddle point separates these two locally attractive solutions. The resident population goes extinct when the saddle point collides with the upper boundary, which leads to a second extinction domain adjacent to the first one. 


\section{Appendix 4 - Dimorphic population}

Here we make use of the general polymorphic equations (A4) and (A10) to describe the population dynamics when there are only two phenotypes, indexed by $x$ and $y$. Deriving a closed dynamical system requires equations for the two global frequencies $p_{x}$ and $p_{y}$ and for the three local frequencies $q_{x \mid x}, q_{x \mid y}$, and $q_{y \mid y}$. Using (A10) and (A5) for the dynamics of local frequencies and (A4) for the dynamics of global frequencies yields

$$
\begin{aligned}
& \frac{d p_{x}(t)}{d t}=\lambda_{x}(t) p_{x}(t) \\
& \frac{d p_{y}(t)}{d t}=\lambda_{y}(t) p_{y}(t) \\
& \frac{d q_{x \mid x}(t)}{d t}=2 \beta_{x}(t) q_{0 \mid x}(t)-\left(2 \delta_{x}(t)+\lambda_{x}(t)\right) q_{x \mid x}(t) \\
& \frac{d q_{x \mid y}(t)}{d t}=\left(\alpha_{x}(t)+\alpha_{y}^{\prime}(t) q_{0 \mid 0}(t)\right) q_{0 \mid y}(t)-\left(\delta_{y}(t)+\delta_{x}(t)+\lambda_{y}(t)\right) q_{x \mid y}(t) \\
& \frac{d q_{y \mid y}(t)}{d t}=2 \beta_{y}(t) q_{0 \mid y}(t)-\left(2 \delta_{y}(t)+\lambda_{y}(t)\right) q_{y \mid y}(t)
\end{aligned}
$$

As pointed out by van Baalen and Rand (1998), the mean-field version of system (A13) with selfishness ( $x$ type) and altruism ( $y$ type) interacting on the lattice is given by

$$
\begin{aligned}
& \frac{d p_{x}}{d t}=\left(\left(b(m)+u_{y}(1-\phi) p_{y}-C(0)\right) p_{0}-d\right) p_{x} \\
& \frac{d p_{y}}{d t}=\left(\left(b(m)+u_{y}(1-\phi) p_{y}-C\left(u_{y}\right)\right) p_{0}-d\right) p_{y}
\end{aligned}
$$

This system readily shows that selfishness is uninvadable because it benefits from the same amount of altruism without paying the costs. This serves to highlight that spatial population heterogeneity is a key prerequisite for the invasion of altruism in a selfish world.

\section{Appendix 5 - Invasion structure and fitness}

Let $\tilde{q}_{0 \mid y}, \tilde{q}_{x \mid y}$, and $\tilde{q}_{y \mid y}$ denote the pseudo-equilibrium local frequencies characterizing the mutant correlation structure during invasion. These terms are the steady states of (A13) when $x$ is a resident type at ecological equilibrium and $y$ is a mutant type at low frequency, or

$$
\begin{aligned}
& \frac{d q_{x \mid y}(t)}{d t}=\left(\bar{\alpha}_{x}+\tilde{\alpha}_{y}^{\prime} \bar{q}_{0 \mid 0}\right) \tilde{q}_{0 \mid y}-\left(\tilde{\delta}_{y}+\bar{\delta}_{x}+\tilde{\lambda}_{y}\right) \tilde{q}_{x \mid y}=0 \\
& \frac{d q_{y \mid y}(t)}{d t}=2 \tilde{\beta}_{y} \tilde{q}_{0 \mid y}-\left(2 \tilde{\delta}_{y}+\tilde{\lambda}_{y}\right) \tilde{q}_{y \mid y}=0
\end{aligned}
$$


Noting that $\tilde{q}_{y \mid 0}=0$ when the mutant is rare, the non-linear system involves three unknowns $\left(\tilde{q}_{0 \mid y}, \tilde{q}_{x \mid y}\right.$, and $\left.\tilde{q}_{y \mid y}\right)$ and two equations, along with the constraint $\tilde{q}_{0 \mid y}=1-\tilde{q}_{x \mid y}-\tilde{q}_{y \mid y}$.

The non-linear system (A15) can be used generically to evaluate numerically the spatial invasion fitness. However, one further analytical step can be taken by introducing the Taylor expansion of spatial invasion fitness up to the first order:

$$
s_{x}(y)=s_{x}(x)+\left.(y-x) \frac{\partial s_{x}(y)}{\partial y}\right|_{y=x}+o(y-x) .
$$

For the degenerate mutant, $y=x$, we can solve analytically the non-linear system (A15) using symbolic resolution (e.g., Mathematica). This yields the solutions $\tilde{q}_{0 \mid y}=\bar{q}_{0 \mid x}$, $\tilde{q}_{y \mid y}=\bar{q}_{y \mid y}$ given as Equation (5) in the text, and $s_{x}(y)=s_{x}(x)=0$. We now consider a slightly perturbed resident investment, i.e., $u_{y}=u_{x}+\varepsilon, \quad \tilde{q}_{0 \mid y}=\bar{q}_{0 \mid x}+a \varepsilon$, and $\tilde{q}_{y \mid y}=\bar{q}_{y \mid y}+b \varepsilon$. The term $a$ measures the marginal gain or loss of open space in a mutant's neighborhood relative to a resident. The term $b$ measures the marginal gain or loss of relatives in a mutant's neighborhood relative to a resident. Plugging these approximations in the spatial invasion fitness defined by (1) yields after some algebra

$$
s_{x}(y)=\varepsilon \bar{q}_{0 \mid x}\left((1-\phi) \bar{q}_{y \mid y}-a\left((1-\phi) u_{x}-\frac{d}{\bar{q}_{0 \mid x}^{2}}\right)-\frac{C\left(u_{y}\right)-C\left(u_{x}\right)}{\varepsilon}\right),
$$

from which we derive the selection derivative (3) at the limit where $\varepsilon$ vanishes. Next, we use the non-linear system (A15) to solve for the $a$ and $b$ terms introduced before and to evaluate numerically the different components of (A17). 